Copyright $@ 2010$ Institute of Electrical and electronics Engineers, Inc.

All Rights reserved.

Personal use of this material, including one hard copy reproduction, is permitted.

Permission to reprint, republish and/or distribute this material in whole or in part for any other purposes must be obtained from the IEEE.

For information on obtaining permission, send an e-mail message to stds-igr@ieee.org.

By choosing to view this document, you agree to all provisions of the copyright laws protecting it.

Individual documents posted on this site may carry slightly different copyright restrictions.

For specific document information, check the copyright notice at the beginning of each document. 


\title{
Fault-Tolerant Master-Slave Synchronization for Lur'e Systems Using Time-Delay Feedback Control
}

\author{
Maiying Zhong and Qing-Long Han
}

\begin{abstract}
This paper deals with fault-tolerant master-slave synchronization for Lur'e systems using time-delay feedback control. Taking a general nature of fault in the master system into account, a new synchronization scheme, namely, fault-tolerant master-slave synchronization, is proposed, by which the master-slave synchronization can be achieved no matter if the fault occurs or not. By making use of an observer-based fault estimator and a modified time-delay feedback controller, the fault-tolerant master-slave synchronization is formulated so as to discuss the global asymptotic stability of the error system and the bound of energy gain from fault to state and fault estimation error vectors. Some new delay-dependent criteria are derived to analyze the synchronization error system, and based on the analysis results, a sufficient condition on the existence of such a master-slave synchronization scheme and a solution to the controller and fault-estimator gain matrices are obtained in terms of linear matrix inequalities. Finally, a Chua's circuit is used to illustrate the effectiveness of the proposed method.
\end{abstract}

Index Terms-Fault estimator, fault tolerance, Lur'e system, master-slave synchronization, time delay.

\section{INTRODUCTION}

C HAOS SYNCHRONIZATION has been a focused research topic during the last decade due to its theoretical importance and practical applications [2], [6], [10], [17], [21]-[25], [28]. Since there are some nonlinear systems, such as Chua's circuit, $n$-scroll attractors, and hyperchaotic attractors, that can be represented as Lur'e systems, the chaotic synchronization problem was reformulated as a Lur'e system, and the absolute stability of its error system was discussed [5], [7], [8], [27]. In particular, due to the propagation delay frequently encountered in the remote master-slave synchronization scheme, recently, there have been some research efforts to investigate

Manuscript received July 14, 2007; revised August 04, 2008. First published November 11, 2008; current version published July 01, 2009. This work was supported in part by the Central Queensland University under the Research Advancement Awards Scheme Project "Robust Fault Detection, Filtering, and Control for Uncertain Systems with Time-Varying Delay" (January 2006-December 2008). The work of M. Zhong was supported in part by the National Nature Science Foundation of China under Grants 60774071 and 60736025 , by the Scientific Research Project of the Education Ministry of China under Grant 20050422036, and by the Shandong Province Project under Grant 2005BS01007. This paper was recommended by Associate Editor C. W. Wu.

M. Zhong is with the School of Instrument Science and Opto-Electronics Engineering, Beihang University, Beijing 100083, China, and also with the Centre for Intelligent and Networked Systems, Central Queensland University, Rockhampton, QLD 4702, Australia.

Q.-L. Han is with the Centre for Intelligent and Networked Systems and the School of Computing Sciences, Faculty of Arts, Business, Informatics and Education, Central Queensland University, Rockhampton, QLD 4702, Australia (e-mail: q.han@cqu.edu.au).

Digital Object Identifier 10.1109/TCSI.2008.2006218 the delay effect on master-slave synchronization [1], [13], [14], [18], [30]. In [30], a constant time-delay static error output feedback control was used for the master-slave synchronization of Lur'e systems, and some delay-independent and -dependent conditions were given. In [18], a feedback control including both static error state feedback and time-delay static output feedback was considered, and some algebraic criteria were derived. In [1], the results in [18] and [30] were generalized and improved. In [13], based on a more general Lur'e-Postnikov Lyapunov functional, some less conservative delay-dependent synchronization criteria for Lur'e systems were obtained and formulated in the form of linear matrix inequalities (LMIs). In [14], the author designed time-varying delay feedback controllers for the master-slave synchronization of Lur'e systems, and some delay-dependent criteria were derived by applying the Lyapunov-Krasovskii functional approach. However, most of the results on master-slave synchronization for Lur'e systems did not consider the influence of a fault. In fact, the occurrence of a fault is usually inevitable in a real system, such as the component failure in a circuit or the changes in model parameters caused by malfunctions in the sensors. In order to increase the reliability and achieve master-slave synchronization with the consideration of a fault, it is of practical significance to develop a fault-tolerant synchronization scheme, which is one motivation of the present study.

On the other hand, fault diagnosis and fault-tolerant control has been an active research topic during the past two decades for increasing the safety and reliability of the considered systems [9], [11]. Particularly, the study of reliable and fault-tolerant controls for time-delay systems has received some attention in recent years [4], [19], [26], [29]. In [26] and [29], the reliable control for linear time-delay systems has been considered using a single controller with a fixed gain. In [19], a model reference adaptive control for a class of linear systems with state delay in the presence of unknown actuator failures has been developed. In [4], an iterative learning observer is considered for fault detection, estimation, and accommodation in a nonlinear time-delay system to offset the effect of a fault on the system stability. To the best of the authors' knowledge, however, research on the problem of fault-tolerant feedback control for Lur'e systems with time delay is still open and remains challenging, which is the another motivation of the current study.

In this paper, we will deal with the problem of fault-tolerant master-slave synchronization for Lur'e systems using time-delay feedback control. A general nature of fault in the master system will be considered, and an observer-based fault estimator will be used to estimate the fault, which includes stepwise, slow-drifting, and sine-wave faults. A new master-slave 
synchronization scheme will be introduced by incorporating the fault estimation into the feedback controller. Then, the problem of fault-tolerant master-slave synchronization will be formulated so as to discuss the global asymptotic stability of the error system and the bound of energy gain from fault to state and fault estimation error vectors. Applying the Lyapunov-Krasovskii approach, the fault-tolerant controller and fault estimator will be designed by solving LMIs. We will use a Chua's circuit to illustrate the effectiveness of the proposed method.

Notations: The superscripts " 1 " and "T" stand for the inverse and transpose of a matrix, respectively. $\mathbb{R}^{n}$ denotes the $n$-dimensional Euclidean space. $\mathbb{R}^{n \times m}$ is the set of all $n \times m$ real matrices. $I$ is the identity matrix with appropriate dimensions. $\mathcal{L}_{2}$ denotes the space of square integrable vector functions over $[0, \infty)$ with norm $\|\cdot\|_{2}=\left(\int_{0}^{\infty}\|\cdot\|^{2} \mathrm{~d} t\right)^{1 / 2}$ or the space of energy bounded signals with energy $\|\cdot\|_{2}^{2}$, where $\|\cdot\|$ denotes the Euclidean vector norm of $(\cdot)$. For a real matrix $P, P>0$ (respectively, $P<0$ ) means that $P$ is real symmetric and positive definite (respectively, negative definite). $\operatorname{diag}(\cdots)$ denotes a block-diagonal matrix. For an arbitrary matrix $W$ and two symmetric matrices $P$ and $Q$, the symmetric term in a symmetric matrix is denoted by ${ }^{*}$, i.e., $\left(\begin{array}{cc}P & W \\ * & Q\end{array}\right)=\left(\begin{array}{cc}P & W \\ W^{\mathrm{T}} & Q\end{array}\right)$. For $t_{0}>0, \delta\left(t-t_{0}\right)$ is an impulse function satisfying $\delta\left(t-t_{0}\right)=$ $0\left(t \neq t_{0}\right)$ and $\int_{0}^{\infty} \delta\left(t-t_{0}\right) \mathrm{d} t=1$.

\section{PRELIMINARIES AND PROBLEM STATEMENT}

Consider the following master-slave synchronization scheme using time-delay static error feedback control [18]:

$$
\begin{array}{cc}
\mathcal{M}_{0}: & \left\{\begin{array}{l}
\dot{x}(t)=A x(t)+B \varphi(C x(t)) \\
z_{x}(t)=D x(t)
\end{array}\right. \\
\mathcal{S}: & \left\{\begin{array}{l}
\dot{y}(t)=A y(t)+B \varphi(C y(t))+u(t) \\
z_{y}(t)=D y(t)
\end{array}\right. \\
\mathcal{C}_{0}: \quad u(t)=-K_{1}(x(t)-y(t)) \\
+H_{1}\left(z_{x}(t-\tau)-z_{y}(t-\tau)\right)
\end{array}
$$

where $\mathcal{M}_{0}, \mathcal{S}$, and $\mathcal{C}_{0}$ denote the master system, slave system, and controller, respectively; $\tau>0$ is the time delay; the master and slave systems are Lur'e systems with state vectors $x(t)$ and $y(t) \in \mathbb{R}^{n}$ and output vectors $z_{x}(t)$ and $z_{y}(t) \in \mathbb{R}^{l}$, respectively; $A, B, C$, and $D$ are constant matrices with appropriate dimensions; $K_{1}$ and $H_{1}$ are controller gain matrices; $\varphi(\cdot): \mathbb{R}^{m} \rightarrow$ $\mathbb{R}^{m}$ is a memoryless nonlinear vector-valued function which is globally Lipschitz, $\varphi(0)=0$, and suppose that the nonlinearity $\varphi(\cdot)$ is time invariant, decoupled, and satisfies a sector condition with $\varphi_{i}(\xi)$ belonging to a sector $[0, k]$, i.e.,

$$
\varphi_{i}(\xi)\left[\varphi_{i}(\xi)-k \xi\right] \leq 0 \quad \forall \xi \in \mathbb{R}, i=1, \ldots, m
$$

Defining $e(t)=x(t)-y(t)$, we have the error system

$$
\dot{e}(t)=\left(A+K_{1}\right) e(t)+H_{1} D e(t-\tau)+B \eta(C e(t), y(t))
$$

where

$$
\eta(C e(t), y(t))=\varphi(C e(t)+C y(t))-\varphi(C y(t))
$$

The initial condition of system (5) is defined as

$$
e(\theta)=\phi(\theta) \quad \forall \theta \in[-\tau, 0]
$$

where $\phi(\theta)$ is a continuous vector-valued function.

The goal in the master-slave synchronization of the system described by (1)-(4) is to ensure the global asymptotic stability of system (5) by choosing controller gain matrices $K_{1}$ and $H_{1}$.

Taking a fault in the master system into account, we have

$$
\mathcal{M}:\left\{\begin{array}{l}
\dot{x}(t)=A x(t)+B \varphi(C x(t))+B_{f} f(t) \\
z_{x}(t)=D x(t)
\end{array}\right.
$$

where $f(t) \in \mathbb{R}^{p}$ is the fault and $B_{f}$ is a constant matrix with compatible dimensions. The error system (5) becomes

$$
\begin{aligned}
\dot{e}(t)=\left(A+K_{1}\right) e(t)+ & H_{1} D e(t-\tau) \\
& +B \eta(C e(t), y(t))+B_{f} f(t) .
\end{aligned}
$$

In this case, it is usually impossible to ensure the asymptotic stability of system (8) when a fault $f(t) \neq 0$ occurs.

In order to find a fault-tolerant controller for master-slave synchronization, we first analyze the characteristics of unknown constant, ramp, and sine-wave faults, respectively.

It is known that a constant but unknown fault can be characterized as

$$
\begin{aligned}
& f(t)= \begin{cases}0, & \text { when } t \leq t_{f} \\
\beta_{1}, & \text { when } t>t_{f}\end{cases} \\
& \left\{\begin{array}{l}
\dot{f}(t)=f_{0}(t) \\
f(0)=0
\end{array}\right.
\end{aligned}
$$

where $f_{0}(t)=\beta_{1} \cdot \delta\left(t-t_{f}\right)$ and $t_{f}$ and $\beta_{1}$ denote the occurring time and value of the fault, respectively.

When a ramp fault is considered, it is assumed that there is an unknown constant $\beta_{2}$ such that $\left|\beta_{2}\right|<1$ and

$$
\begin{cases}f(t)=0, & \text { when } t \leq t_{f} \\ \dot{f}(t)=\beta_{2}, & \text { when } t>t_{f}\end{cases}
$$

where $t_{f}$ is the occurring time of the fault, $\beta_{2}$ is the slope of the ramp fault, and both $t_{f}$ and $\beta_{2}$ are unknown. For this case, the fault can also be regarded as the output of the following system with a fictitious signal $f_{0}(t)=\beta_{2} \cdot \delta\left(t-\tau_{f}\right)$ :

$$
\left\{\begin{array}{l}
\dot{x}_{f}(t)=\left(\begin{array}{ll}
0 & 0 \\
1 & 0
\end{array}\right) x_{f}(t)+\left(\begin{array}{l}
1 \\
0
\end{array}\right) f_{0}(t) \\
f(t)=\left(\begin{array}{ll}
0 & 1
\end{array}\right) x_{f}(t) \\
x_{f}(0)=0
\end{array}\right.
$$

where $x_{f}(t) \in \mathbb{R}^{2}$. 
For a sine-wave fault with a given frequency $\omega$ but unknown phase $b$, amplitude $\beta_{3}$, and occurring time $\tau_{f}$, it can be the output of the following system:

$$
\left\{\begin{array}{l}
\dot{x}_{f}(t)=\left(\begin{array}{cc}
0 & -\omega^{2} \\
1 & 0
\end{array}\right) x_{f}(t)+\left(\begin{array}{cc}
\omega & 0 \\
0 & 1
\end{array}\right) f_{0}(t) \\
f(t)=\left(\begin{array}{ll}
0 & 1
\end{array}\right) x_{f}(t) \\
x_{f}(0)=0
\end{array}\right.
$$

where $x_{f}(t) \in \mathbb{R}^{2}$ and $f_{0}(t)=\left(\begin{array}{c}\omega \beta_{3} \cos b \\ \beta_{3} \sin b\end{array}\right) \cdot \delta\left(t-\tau_{f}\right)$.

In this paper, we focus on a more general case. It is assumed that the fault is the output of the following system:

$$
\left\{\begin{array}{l}
\dot{x}_{f}(t)=A_{W f} x_{f}(t)+B_{W f} f_{0}(t) \\
f(t)=C_{W f} x_{f}(t) \\
x_{f}(0)=0
\end{array}\right.
$$

where $x_{f}(t) \in \mathbb{R}^{n_{f}}$ is a state; $A_{W f}, B_{W f}$, and $C_{W f}$ are matrices obtained from the prior knowledge about the possible fault; $f_{0}(t)=\sum_{i=1}^{N} \rho_{i} \delta\left(t-t_{f i}\right), t_{f i}$ and $\rho_{i}$ are unknown parameters, and $N$ is a positive unknown integer. Obviously, systems (9)-(11) are three particular cases of (12) and can be used to model stepwise, slow-drifting, and sine-wave faults, respectively, when $f_{0}(t)=\sum_{i=1}^{N} \rho_{i} \delta\left(t-t_{f i}\right)$.

For the purpose of synchronization, we now consider a modified feedback controller

$$
\begin{aligned}
\mathcal{C}: u(t)=B_{f} \hat{f}(t)-K_{1} & (x(t)-y(t)) \\
& +H_{1}\left(z_{x}(t-\tau)-z_{y}(t-\tau)\right)
\end{aligned}
$$

where $\hat{f}(t)$ is the estimation of fault $f(t)$ given by the following observer-based fault estimator:

$$
\mathcal{F}:\left\{\begin{array}{l}
\dot{\hat{x}}_{f}(t)=A_{W f} \hat{x}_{f}(t)+v(t) \\
v(t)=-K_{2}(x(t)-y(t)) \\
\quad+H_{2}\left(z_{x}(t-\tau)-z_{y}(t-\tau)\right) \\
\hat{f}(t)=C_{W f} \hat{x}_{f}(t) .
\end{array}\right.
$$

$\mathrm{K}_{2}$ and $\mathrm{H}_{2}$ are fault-estimator gain matrices to be determined. Defining

$$
\begin{aligned}
e(t) & =x(t)-y(t) \\
e_{f}(t) & =x_{f}(t)-\hat{x}_{f}(t) \\
r(t) & =f(t)-\hat{f}(t)
\end{aligned}
$$

it follows from (2), (7), (12)-(14) that

$$
\left\{\begin{aligned}
\dot{e}(t)= & \left(A+K_{1}\right) e(t)-H_{1} D e(t-\tau)+B_{f} C_{W f} e_{f}(t) \\
& +B \eta(C e(t), y(t)) \\
\dot{e}_{f}(t)= & A_{W f} e_{f}(t)+K_{2} e(t)-H_{2} D e(t-\tau) \\
& +B_{W f} f_{0}(t) \\
r(t)= & C_{W f} e_{f}(t) \\
e(\theta)= & \phi(\theta) \quad e_{f}(\theta)=0 \quad \forall \theta \in[-\tau, 0]
\end{aligned}\right.
$$

where

$$
\eta(C e(t), y(t))=\varphi(C e(t)+C y(t))-\varphi(C y(t)) .
$$

Let $C^{\mathrm{T}}=\left(\begin{array}{llll}c_{1} & c_{2} & \cdots & c_{m}\end{array}\right), c_{i} \in \mathbb{R}^{n}, i=1,2, \ldots, m$. Suppose that $\eta(C e(t), y(t))$ belongs to the sector $[0, k]$, i.e.,

$$
\begin{aligned}
\eta_{i}\left(c_{i}^{\mathrm{T}} e(t), y(t)\right)\left[\eta_{i}\left(c_{i}^{\mathrm{T}} e(t), y(t)\right)-k c_{i}^{\mathrm{T}} e(t)\right] \leq 0 \\
\forall t \geq 0 .
\end{aligned}
$$

Notice that, if system (15) with $f_{0}(t)=0$ is globally asymptotically stable, then $\lim _{t \rightarrow \infty} e(t)=0$ and $\lim _{t \rightarrow \infty} e_{f}(t)=0$ can be achieved for $f_{0}(t)=\sum_{i=1}^{N} \rho_{i} \delta\left(t-t_{f i}\right)$, which means that the master-slave system is synchronized when a fault characterized by (12) occurs. Therefore, the main objective of fault-tolerant master-slave synchronization is to find matrices $K_{i}$ and $H_{i}(i=1,2)$ such that system(15) with $f_{0}(t)=0$ is globally asymptotically stable. Moreover, we define the following error energy as a cost function to measure the influence of fault on transient state error $e(t)$ and fault estimation error $r(t)$ :

$$
J=\int_{0}^{\infty}\left[e^{\mathrm{T}}(t) M_{1} e(t)+r^{\mathrm{T}}(t) M_{2} r(t)\right] \mathrm{d} t
$$

where $M_{1} \geq 0$ and $M_{2} \geq 0$ are weighting matrices. Introducing a new variable $z_{e}(t)$ as

$$
z_{e}(t)=F\left(\begin{array}{c}
e(t) \\
e_{f}(t)
\end{array}\right)
$$

where $F^{\mathrm{T}} F=\operatorname{diag}\left(M_{1}, C_{W f}^{\mathrm{T}} M_{2} C_{W f}\right)$, one obtains

$$
J=\int_{0}^{\infty} z_{e}^{\mathrm{T}}(t) z_{e}(t) \mathrm{d} t=\left\|z_{e}(t)\right\|_{2}^{2} .
$$

Let

$$
\begin{aligned}
\xi(t) & =\left(\begin{array}{c}
e(t) \\
e_{f}(t)
\end{array}\right) & A_{\xi} & =\left(\begin{array}{cc}
A & B_{f} C_{W f} \\
0 & A_{W f}
\end{array}\right) \\
B_{\xi} & =\left(\begin{array}{c}
B \\
0
\end{array}\right) & B_{\xi f} & =\left(\begin{array}{c}
0 \\
B_{W f}
\end{array}\right) \\
K_{\xi} & =\left(\begin{array}{c}
K_{1} \\
K_{2}
\end{array}\right) & H_{\xi} & =\left(\begin{array}{c}
H_{1} \\
H_{2}
\end{array}\right) \\
D_{\xi} & =\left(\begin{array}{ll}
D & 0
\end{array}\right) & I_{0} & =\left(\begin{array}{ll}
I & 0
\end{array}\right) .
\end{aligned}
$$

We have

$$
\left\{\begin{aligned}
\dot{\xi}(t)= & \left(A_{\xi}+K_{\xi} I_{0}\right) \xi(t)-H_{\xi} D_{\xi} \xi(t-\tau) \\
& +B_{\xi} \eta(C e(t), y(t))+B_{\xi f} f_{0}(t) \\
z_{e}(t)= & F \xi(t) \quad \\
\xi(\theta)= & \phi_{\xi}(\theta) \quad \forall \theta \in[-\tau, 0]
\end{aligned}\right.
$$

where $\phi_{\xi}^{\mathrm{T}}(\theta)=\left(\begin{array}{ll}\phi^{\mathrm{T}}(\theta) & 0\end{array}\right)$.

Now, the problem of fault-tolerant master-slave synchronization can be formulated so as to find matrices $K_{i}$ and $H_{i}(i=$ $1,2)$ such that system $(18)$ is globally asymptotically stable with an energy-gain bound $\gamma^{2}$ for a prescribed $\gamma>0$, i.e., system (18) with $f_{0}(t)=0$ is globally asymptotically stable, and for all $f_{0}(t) \in \mathcal{L}_{2}[0, \infty)$, the performance $\left\|z_{e}(t)\right\|_{2} \leq \gamma\left\|f_{0}(t)\right\|_{2}$ is satisfied under zero initial condition.

The following lemmas are useful in deriving synchronization criteria. 
Lemma 1 [15]: For any constant matrix $M \in \mathbb{R}^{n \times n}, M=$ $M^{\mathrm{T}}>0$, scalar $\tau>0$, and vector function $\dot{x}:[-\tau, 0] \rightarrow \mathbb{R}^{n}$ such that the following integration is well defined:

$$
\begin{aligned}
& -\tau \int_{-\tau}^{0} \dot{x}^{\mathrm{T}}(t+s) M \dot{x}(t+s) \mathrm{d} s \\
& \quad \leq\left(\begin{array}{c}
x(t) \\
x(t-\tau)
\end{array}\right)^{\mathrm{T}}\left(\begin{array}{cc}
-M & M \\
M & -M
\end{array}\right)\left(\begin{array}{c}
x(t) \\
x(t-\tau)
\end{array}\right) .
\end{aligned}
$$

Lemma 2 [12]: For any real matrices $X$ and $Y$ and real symmetric positive definite matrix $R$ with compatible dimensions

$$
X Y+Y^{\mathrm{T}} X^{\mathrm{T}} \leq X R X^{\mathrm{T}}+Y^{\mathrm{T}} R^{-1} Y .
$$

\section{MAIN RESULTS}

In order to design matrices $K_{i}$ and $H_{i}(i=1,2)$, we first need the analysis result on system (18). Choose a Lyapunov-Kravoskii functional candidate as

$$
\begin{aligned}
V\left(t, \xi_{t}\right)=\xi^{\mathrm{T}}(t) P \xi(t) & +\int_{t-\tau}^{t} \xi^{\mathrm{T}}(s) Q \xi(s) \mathrm{d} s \\
& +\int_{t-\tau}^{t}(\tau-t+s) \dot{\xi}^{\mathrm{T}}(s)(\tau R) \dot{\xi}(s) \mathrm{d} s
\end{aligned}
$$

where $\xi_{t}$ is defined as $\xi_{t}=\xi(t+\theta) \forall \theta \in[-\tau, 0]$, and $P, Q$, and $R$ are real $\left(n+n_{f}\right) \times\left(n+n_{f}\right)$ symmetric positive definite matrices. Using (19) and Lemma 1, we have the following result.

Proposition 1: For the given scalars $\gamma>0$ and $\tau>0$ and matrices $K_{\xi}$ and $H_{\xi}$, system (18) is globally asymptotically stable with an energy-gain bound $\gamma^{2}$ if there exist real $\left(n+n_{f}\right) \times\left(n+n_{f}\right)$ matrices $P>0, Q>0$, and $R>0$ and a matrix $\Lambda=\operatorname{diag}\left(\lambda_{1}, \lambda_{2}, \ldots, \lambda_{m}\right)>0$ such that

$$
\left(\begin{array}{cccccc}
(1,1) & (1,2) & (1,3) & P B_{\xi f} & F^{\mathrm{T}} & (1,6) \\
* & (2,2) & 0 & 0 & 0 & (2,6) \\
* & * & -2 \Lambda & 0 & 0 & (3,6) \\
* & * & * & -\gamma^{2} I & 0 & (4,6) \\
* & * & * & * & -I & 0 \\
* & * & * & * & 0 & -R
\end{array}\right)<0
$$

where

$$
\begin{aligned}
& (1,1)=P\left(A_{\xi}+K_{\xi} I_{0}\right)+\left(A_{\xi}+K_{\xi} I_{0}\right)^{\mathrm{T}} P+Q-R \\
& (1,2)=-P H_{\xi} D_{\xi}+R \\
& (2,2)=-Q-R \\
& (1,3)=P B_{\xi}+k C_{\xi}^{\mathrm{T}} \Lambda \\
& (1,6)=\tau\left(A_{\xi}+K_{\xi} I_{0}\right)^{\mathrm{T}} R \\
& (2,6)=-\tau\left(H_{\xi} D_{\xi}\right)^{\mathrm{T}} R \\
& (3,6)=\tau B_{\xi}^{\mathrm{T}} R \\
& (4,6)=\tau B_{\xi f}^{\mathrm{T}} R .
\end{aligned}
$$

Proof: Taking the derivative of $V\left(t, \xi_{t}\right)$ with respect to $t$ along the trajectory of (18) yields

$$
\begin{aligned}
\dot{V}\left(t, \xi_{t}\right)= & \xi^{\mathrm{T}}(t)\left[P\left(A_{\xi}+K_{\xi} I_{0}\right)\right. \\
& \left.+\left(A_{\xi}+K_{\xi} I_{0}\right)^{\mathrm{T}} P+Q\right] \xi(t) \\
& -2 \xi^{\mathrm{T}}(t) P H_{\xi} D_{\xi} \xi(t-\tau) \\
& -\xi^{\mathrm{T}}(t-\tau) Q \xi(t-\tau) \\
& +2 \xi^{\mathrm{T}}(t) P B_{\xi} \eta(C e(t), y(t)) \\
& +2 \xi^{\mathrm{T}}(t) P B_{\xi f} f_{0}(t)+\dot{\xi}^{\mathrm{T}}(t)\left(\tau^{2} R\right) \dot{\xi}(t) \\
& -\int_{t-\tau}^{t} \dot{\xi}^{\mathrm{T}}(s)(\tau R) \dot{\xi}(s) \mathrm{d} s .
\end{aligned}
$$

For any $\Lambda=\operatorname{diag}\left(\lambda_{1}, \lambda_{2}, \ldots, \lambda_{m}\right)>0$, from (16) and (21), we have

$$
\begin{aligned}
\dot{V}\left(t, \xi_{t}\right) \leq & \xi^{\mathrm{T}}(t)\left[P\left(A_{\xi}+K_{\xi} I_{0}\right)\right. \\
& \left.+\left(A_{\xi}+K_{\xi} I_{0}\right)^{\mathrm{T}} P+Q\right] \xi(t) \\
& -2 \xi^{\mathrm{T}}(t) P H_{\xi} D_{\xi} \xi(t-\tau) \\
& -\xi^{\mathrm{T}}(t-\tau) Q \xi(t-\tau) \\
& +2 \xi^{\mathrm{T}}(t) P B_{\xi} \eta(C e(t), y(t)) \\
& +2 \xi^{\mathrm{T}}(t) P B_{\xi f} f_{0}(t)+\dot{\xi}^{\mathrm{T}}(t)\left(\tau^{2} R\right) \dot{\xi}(t) \\
& -2 \sum_{i=1}^{m} \lambda_{i} \eta_{i}\left(c_{i}^{\mathrm{T}} e(t), y(t)\right) \\
& \times\left[\eta_{i}\left(c_{i}^{\mathrm{T}} e(t), y(t)\right)-k c_{i}^{\mathrm{T}} e(t)\right] \\
& -\int_{t-\tau}^{t} \dot{\xi}^{\mathrm{T}}(s)(\tau R) \dot{\xi}(s) \mathrm{d} s .
\end{aligned}
$$

Use Lemma 1 to obtain

$$
\begin{aligned}
& -\int_{t-\tau}^{t} \dot{\xi}^{\mathrm{T}}(s)(\tau R) \dot{\xi}(s) \mathrm{d} s \\
& \qquad\left(\begin{array}{c}
\xi(t) \\
\xi(t-\tau)
\end{array}\right)^{\mathrm{T}}\left(\begin{array}{cc}
-R & R \\
R & -R
\end{array}\right)\left(\begin{array}{c}
\xi(t) \\
\xi(t-\tau)
\end{array}\right) .
\end{aligned}
$$

It follows from (22) that

$$
\begin{aligned}
& \dot{V}\left(t, \xi_{t}\right) \leq \xi^{\mathrm{T}}(t)\left[P\left(A_{\xi}+K_{\xi} I_{0}\right)\right. \\
& \left.+\left(A_{\xi}+K_{\xi} I_{0}\right)^{\mathrm{T}} P+Q\right] \xi(t) \\
& -2 \xi^{\mathrm{T}}(t) P H_{\xi} D_{\xi} \xi(t-\tau) \\
& -\xi^{\mathrm{T}}(t-\tau) Q \xi(t-\tau) \\
& +2 \xi^{\mathrm{T}}(t) P B_{\xi} \eta(C e(t), y(t)) \\
& +2 \xi^{\mathrm{T}}(t) P B_{\xi f} f_{0}(t)+\dot{\xi}^{\mathrm{T}}(t)\left(\tau^{2} R\right) \dot{\xi}(t) \\
& +\left(\xi^{\mathrm{T}}(t) \quad \xi^{\mathrm{T}}(t-\tau)\right) \\
& \times\left(\begin{array}{cc}
-R & R \\
R & -R
\end{array}\right)\left(\begin{array}{c}
\xi(t) \\
\xi(t-\tau)
\end{array}\right) \\
& -2 \eta^{\mathrm{T}}(C e(t), y(t)) \Lambda \eta(C e(t), y(t)) \\
& +2 k \eta^{\mathrm{T}}(C e(t), y(t)) \Lambda C_{\xi} \xi(t)
\end{aligned}
$$

where $C_{\xi}=\left(\begin{array}{ll}C & 0\end{array}\right)$. 
First, we analyze the global asymptotic stability of system (18) with $f_{0}(t)=0$. In the case of $f_{0}(t)=0$, from (23), one obtains

$$
\dot{V}\left(t, \xi_{t}\right) \leq \varsigma_{1}^{\mathrm{T}}(t) \Theta \varsigma_{1}(t)
$$

where

$$
\begin{aligned}
& \varsigma_{1}^{\mathrm{T}}(t)=\left(\xi^{\mathrm{T}}(t) \quad \xi^{\mathrm{T}}(t-\tau) \quad \eta^{\mathrm{T}}(C e(t), y(t))\right) \\
& \Theta=\left(\begin{array}{ccc}
(1,1) & (1,2) & (1,3) \\
* & (2,2) & -\left(H_{\xi} D_{\xi}\right)^{\mathrm{T}}\left(\tau^{2} R\right) B_{\xi} \\
* & * & -2 \Lambda+B_{\xi}^{\mathrm{T}}\left(\tau^{2} R\right) B_{\xi}
\end{array}\right)
\end{aligned}
$$

with

$$
\begin{aligned}
(1,1)= & P\left(A_{\xi}+K_{\xi} I_{0}\right)+\left(A_{\xi}+K_{\xi} I_{0}\right)^{\mathrm{T}} P+Q-R \\
& +\left(A_{\xi}+K_{\xi} I_{0}\right)^{\mathrm{T}}\left(\tau^{2} R\right)\left(A_{\xi}+K_{\xi} I_{0}\right) \\
(1,2)= & -P H_{\xi} D_{\xi}+R-\left(A_{\xi}+K_{\xi} I_{0}\right)^{\mathrm{T}}\left(\tau^{2} R\right) H_{\xi} D_{\xi} \\
(1,3)= & P B_{\xi}+k C_{\xi}^{\mathrm{T}} \Lambda+\left(A_{\xi}+K_{\xi} I_{0}\right)^{\mathrm{T}}\left(\tau^{2} R\right) B_{\xi} \\
(2,2)= & -Q-R+\left(H_{\xi} D_{\xi}\right)^{\mathrm{T}}\left(\tau^{2} R\right) H_{\xi} D_{\xi} .
\end{aligned}
$$

In view of Schur complement, LMI (20) implies that $\Theta<0$. Then, we have $\dot{V}\left(t, \xi_{t}\right)<0$ for all $\varsigma_{1}(t) \neq 0$, from which we conclude that system (18) with $f_{0}(t)=0$ is globally asymptotically stable.

Next, we consider performance $\left\|z_{e}(t)\right\|_{2} \leq \gamma\left\|f_{0}(t)\right\|_{2}$ for all $f_{0}(t) \in \mathcal{L}_{2}[0, \infty)$ and a prescribed $\gamma>0$ under condition $\phi(\theta)=0 \forall \theta \in[-\tau, 0]$. Define

$$
J_{f}=\int_{0}^{\infty}\left[z_{e}^{\mathrm{T}}(t) z_{e}(t)-\gamma^{2} f_{0}^{\mathrm{T}}(t) f_{0}(t)\right] \mathrm{d} t .
$$

From (23) and (24), we have

$$
\begin{aligned}
& J_{f}=\int_{0}^{\infty}\left[z_{e}^{\mathrm{T}}(t) z_{e}(t)-\gamma^{2} f_{0}^{\mathrm{T}}(t) f_{0}(t)+\dot{V}\left(t, \xi_{t}\right)\right] \mathrm{d} t \\
&-\left.V\left(t, \xi_{t}\right)\right|_{t \rightarrow \infty}+\left.V\left(t, \xi_{t}\right)\right|_{t=0} \\
& \leq \int_{0}^{\infty}\left\{\xi ^ { \mathrm { T } } ( t ) \left[P\left(A_{\xi}+K_{\xi} I_{0}\right)+\left(A_{\xi}+K_{\xi} I_{0}\right)^{\mathrm{T}} P\right.\right. \\
&\left.+Q+F^{\mathrm{T}} F\right] \xi(t) \\
&-2 \xi^{\mathrm{T}}(t) P H_{\xi} D_{\xi} \xi(t-\tau) \\
&-\xi^{\mathrm{T}}(t-\tau) Q \xi(t-\tau) \\
&+2 \xi^{\mathrm{T}}(t) P B_{\xi} \eta(C e(t), y(t)) \\
&+2 \xi^{\mathrm{T}}(t) P B_{\xi f} f_{0}(t)+\dot{\xi}^{\mathrm{T}}(t)\left(\tau^{2} R\right) \dot{\xi}(t) \\
&+\left(\xi^{\mathrm{T}}(t) \quad \xi^{\mathrm{T}}(t-\tau)\right) \\
& \quad \times\left(\begin{array}{cc}
-R & R \\
R & -R
\end{array}\right)\left(\begin{array}{c}
\xi(t) \\
\xi(t-\tau)
\end{array}\right) \\
&-2 \eta^{\mathrm{T}}(C e(t), y(t)) \Lambda \eta(C e(t), y(t)) \\
&\left.+2 k \eta^{\mathrm{T}}(C e(t), y(t)) \Lambda C_{\xi} \xi(t)-\gamma^{2} f_{0}^{\mathrm{T}}(t) f_{0}(t)\right\} \mathrm{d} t \\
&-\left.V\left(t, \xi_{t}\right)\right|_{t \rightarrow \infty}+\left.V\left(t, \xi_{t}\right)\right|_{t=0} .
\end{aligned}
$$

With the observation of

$$
\left.V\left(t, \xi_{t}\right)\right|_{t \rightarrow \infty} \geq\left. 0 \quad V\left(t, \xi_{t}\right)\right|_{t=0}=0
$$

we then have

$$
J_{f} \leq \int_{0}^{\infty} \varsigma_{2}^{\mathrm{T}}(t) \Psi \varsigma_{2}(t) \mathrm{d} t
$$

where

$$
\begin{aligned}
& \varsigma_{2}^{\mathrm{T}}(t)=\left(\begin{array}{llll}
\xi^{\mathrm{T}}(t) & \xi^{\mathrm{T}}(t-\tau) & \eta^{\mathrm{T}}(C e(t), y(t)) & \left.f_{0}^{\mathrm{T}}(t)\right)
\end{array}\right. \\
& \Psi=\left(\begin{array}{cccc}
(1,1) & (1,2) & (1,3) & (1,4) \\
* & (2,2) & (2,3) & (2,4) \\
* & * & (3,3) & (3,4) \\
* & * & * & (4,4)
\end{array}\right)
\end{aligned}
$$

with

$$
\begin{aligned}
(1,1)= & P\left(A_{\xi}+K_{\xi} I_{0}\right)+\left(A_{\xi}+K_{\xi} I_{0}\right)^{\mathrm{T}} P+F^{\mathrm{T}} F+Q \\
& -R+\left(A_{\xi}+K_{\xi} I_{0}\right)^{\mathrm{T}}\left(\tau^{2} R\right)\left(A_{\xi}+K_{\xi} I_{0}\right) \\
(1,2)= & -P H_{\xi} D_{\xi}+R-\left(A_{\xi}+K_{\xi} I_{0}\right)^{\mathrm{T}}\left(\tau^{2} R\right) H_{\xi} D_{\xi} \\
(1,3)= & P B_{\xi}+k C_{\xi}^{\mathrm{T}} \Lambda+\left(A_{\xi}+K_{\xi} I_{0}\right)^{\mathrm{T}}\left(\tau^{2} R\right) B_{\xi} \\
(1,4)= & P B_{\xi f}+\left(A_{\xi}+K_{\xi} I_{0}\right)^{\mathrm{T}}\left(\tau^{2} R\right) B_{\xi f} \\
(2,2)= & -Q-R+\left(H_{\xi} D_{\xi}\right)^{\mathrm{T}}\left(\tau^{2} R\right) H_{\xi} D_{\xi} \\
(2,3)= & -\left(H_{\xi} D_{\xi}\right)^{\mathrm{T}}\left(\tau^{2} R\right) B_{\xi} \\
(2,4)= & -\left(H_{\xi} D_{\xi}\right)^{\mathrm{T}}\left(\tau^{2} R\right) B_{\xi f} \\
(3,3)= & -2 \Lambda+B_{\xi}^{\mathrm{T}}\left(\tau^{2} R\right) B_{\xi} \\
(3,4)= & B_{\xi}^{\mathrm{T}}\left(\tau^{2} R\right) B_{\xi f} \\
(4,4)= & -\gamma^{2} I+B_{\xi f}^{\mathrm{T}}\left(\tau^{2} R\right) B_{\xi f} .
\end{aligned}
$$

Using Schur complement, we have $\Psi<0$ from LMI (20). Thus, $J_{f} \leq 0$, which implies that $\left\|z_{e}(t)\right\|_{2} \leq \gamma\left\|f_{0}(t)\right\|_{2}$. This completes the proof.

When the third term is ignored, the Lyapunov-Kravoskii functional candidate (19) becomes

$$
V_{1}\left(t, \xi_{t}\right)=\xi^{\mathrm{T}}(t) P \xi(t)+\int_{t-\tau}^{t} \xi^{\mathrm{T}}(s) Q \xi(s) \mathrm{d} s .
$$

Then, Proposition 1 implies the following delay-independent result.

Corollary 1: For a given scalar $\gamma>0$ and matrices $K_{\xi}$ and $H_{\xi}$, system (18) is globally asymptotically stable with an energy-gain bound $\gamma^{2}$ if there exist real $\left(n+n_{f}\right) \times\left(n+n_{f}\right)$ matrices $P>0$ and $Q>0$ and a matrix $\Lambda=\operatorname{diag}\left(\lambda_{1}, \lambda_{2}, \ldots, \lambda_{m}\right)>0$ such that

$$
\left(\begin{array}{ccccc}
(1,1) & -P H_{\xi} D_{\xi} & (1,3) & P B_{\xi f} & F^{\mathrm{T}} \\
* & -Q & 0 & 0 & 0 \\
* & * & -2 \Lambda & 0 & 0 \\
* & * & * & -\gamma^{2} I & 0 \\
* & * & * & * & -I
\end{array}\right)<0
$$

where

$$
\begin{aligned}
& (1,1)=P\left(A_{\xi}+K_{\xi} I_{0}\right)+\left(A_{\xi}+K_{\xi} I_{0}\right)^{\mathrm{T}} P+Q \\
& (1,3)=P B_{\xi}+k C_{\xi}^{\mathrm{T}} \Lambda .
\end{aligned}
$$

If the fault $f(t)$ is not taken into account, the master-slave synchronization is formulated as the global asymptotic stability of error system (5). Setting $P=\operatorname{diag}\left(P_{1}, 0\right), Q=\operatorname{diag}\left(Q_{1}, 0\right)$, 


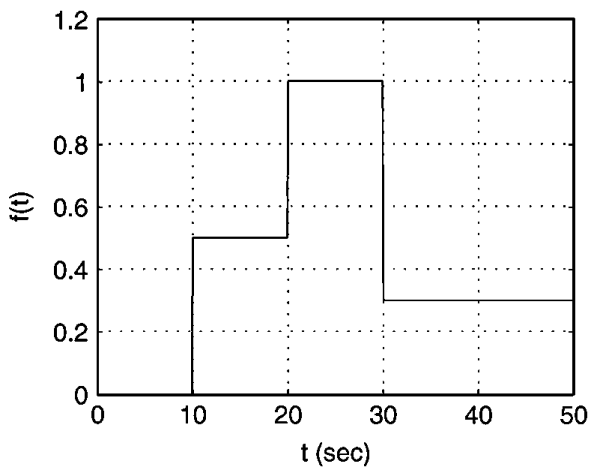

Fig. 1. Stepwise fault signal $f(t)$.

and $R=\operatorname{diag}\left(R_{1}, 0\right)$, where $P_{1}, Q_{1}$, and $R_{1}$ are $n \times n$ real positive definite matrices, the Lyapunov-Kravoskii functional candidate (19) is reduced to

$$
\begin{aligned}
V_{2}\left(t, e_{t}\right)=e^{\mathrm{T}}(t) P_{1} e(t) & +\int_{t-\tau}^{t} e^{\mathrm{T}}(s) Q_{1} e(s) \mathrm{d} s \\
& +\int_{t-\tau}^{t}(\tau-t+s) \dot{e}^{\mathrm{T}}(s)\left(\tau R_{1}\right) \dot{e}(s) \mathrm{d} s .
\end{aligned}
$$

Then, Proposition 1 implies the synchronization criterion Corollary 3 in [14].

Corollary 2: For the given scalars $\gamma>0$ and $\tau>0$ and matrices $K_{1}$ and $H_{1}$, system (18) is globally asymptotically stable if there exist real $n \times n$ matrices $P_{1}>0, Q_{1}>0$ and $R_{1}>0$ and a matrix $\Lambda_{1}=\operatorname{diag}\left(\lambda_{11}, \lambda_{12}, \ldots, \lambda_{1 m}\right)>0$ such that

$$
\left(\begin{array}{cccc}
(1,1) & (1,2) & (1,3) & \tau\left(A+K_{1}\right)^{\mathrm{T}} R_{1} \\
* & (2,2) & 0 & -\tau\left(H_{1} D\right)^{\mathrm{T}} R_{1} \\
* & * & -2 \Lambda_{1} & \tau B^{\mathrm{T}} R_{1} \\
* & * & * & -R_{1}
\end{array}\right)<0
$$

where

$$
\begin{aligned}
& (1,1)=P_{1}\left(A+K_{1}\right)+\left(A+K_{1}\right)^{\mathrm{T}} P_{1}+Q_{1}-R_{1} \\
& (1,2)=-P_{1} H_{1} D+R_{1} \\
& (1,3)=P B+k C^{\mathrm{T}} \Lambda_{1} \\
& (2,2)=-Q_{1}-R_{1} .
\end{aligned}
$$

We are now in the position to design a feedback controller and fault estimator for the fault-tolerant master-slave synchronization. Applying Proposition 1, pre- and post-multiplying both sides of (20) with $\operatorname{diag}\left(I, I, I, I, I, P R^{-1}\right)$ and its transpose, and letting

$$
Y_{K}=P K_{\xi} \quad Y_{H}=P H_{\xi}
$$

it is easy to see that system (18) is globally asymptotically stable with an energy-gain bound $\gamma^{2}$ if there exist real $\left(n+n_{f}\right) \times$ $\left(n+n_{f}\right)$ matrices $P>0, Q>0$, and $R>0$, a matrix
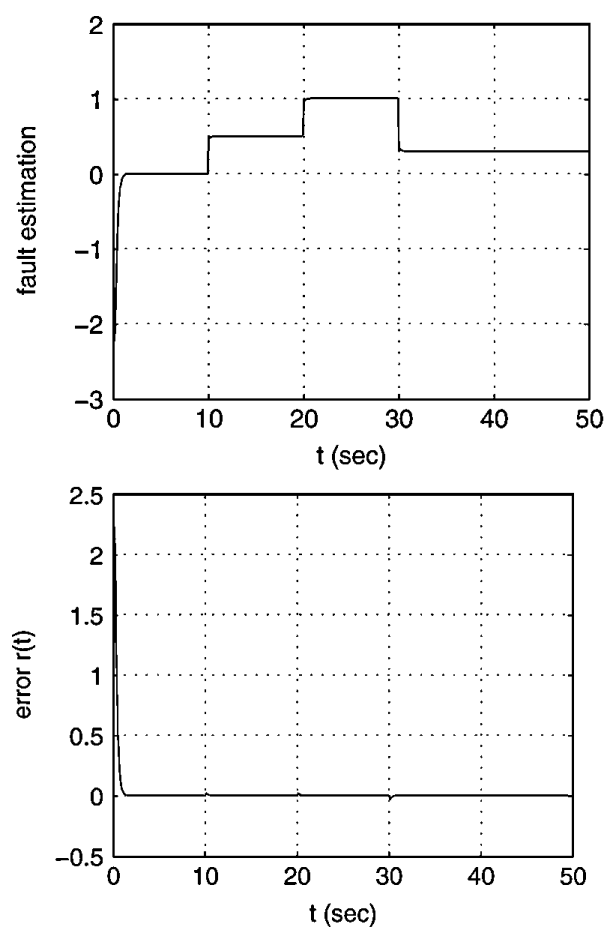

Fig. 2. Fault estimation $\hat{f}(t)$ and estimation error $r(t)$.

$\Lambda=\operatorname{diag}\left(\lambda_{1}, \lambda_{2}, \ldots, \lambda_{m}\right)>0$, and matrices $Y_{K}$ and $Y_{H}$ with appropriate dimensions such that

$$
\left(\begin{array}{cccccc}
(1,1) & (1,2) & (1,3) & (1,4) & F^{\mathrm{T}} & (1,6) \\
* & (2,2) & 0 & 0 & 0 & (2,6) \\
* & * & -2 \Lambda & 0 & 0 & (3,6) \\
* & * & * & -\gamma^{2} I & 0 & (4,6) \\
* & * & * & * & -I & 0 \\
* & * & * & * & * & (6,6)
\end{array}\right)<0
$$

where

$$
\begin{aligned}
& (1,1)=A_{\xi}^{\mathrm{T}} P+P A_{\xi}+Y_{K} I_{0}+I_{0}^{\mathrm{T}} Y_{K}^{\mathrm{T}}+Q-R \\
& (1,2)=-Y_{H} D_{\xi}+R \\
& (1,3)=P B_{\xi}+k C_{\xi}^{\mathrm{T}} \Lambda \\
& (1,4)=P B_{\xi} \\
& (1,6)=\tau\left(A_{\xi}^{\mathrm{T}} P+I_{0}^{\mathrm{T}} Y_{K}^{\mathrm{T}}\right) \\
& (2,2)=-Q-R \\
& (2,6)=-\tau D_{\xi}^{\mathrm{T}} Y_{H}^{\mathrm{T}} \\
& (3,6)=\tau B_{\xi}^{\mathrm{T}} P \\
& (4,6)=\tau B_{\xi f}^{\mathrm{T}} P \\
& (6,6)=-P R^{-1} P .
\end{aligned}
$$

Notice that condition (26) includes nonlinear term $-P R^{-1} P$. Using Lemma 2, for any scalar $\varepsilon>0$, we have

$$
-P R^{-1} P \leq-2 \varepsilon P+\varepsilon^{2} R .
$$



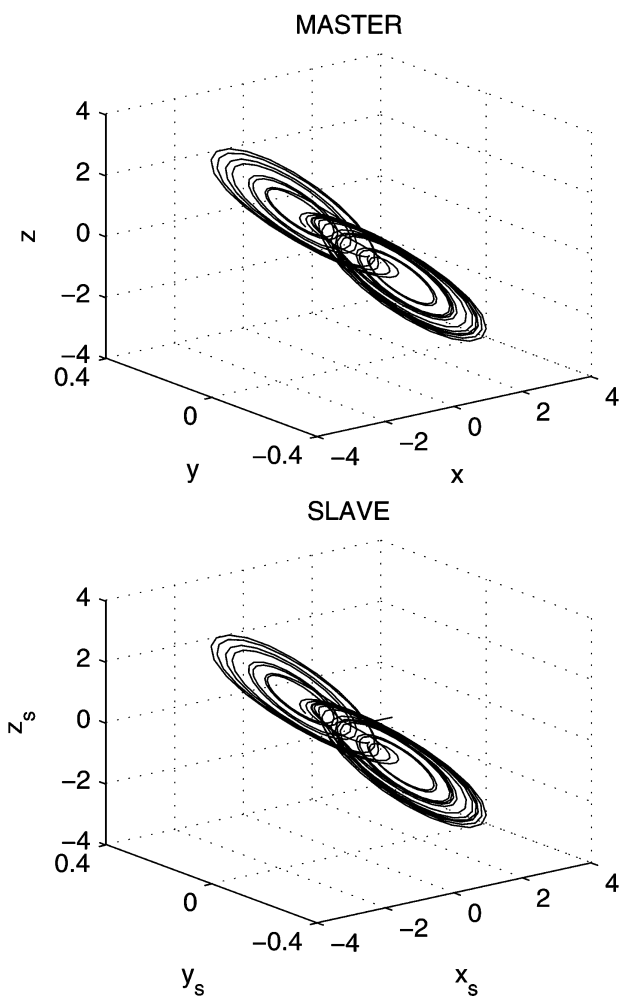

Fig. 3. Master-slave state without fault.

In light of Schur complement, we have (26) from (27) and

$$
\left(\begin{array}{ccccccc}
(1,1) & (1,2) & (1,3) & (1,4) & F^{\mathrm{T}} & (1,6) & 0 \\
* & (2,2) & 0 & 0 & 0 & (2,6) & 0 \\
* & * & -2 \Lambda & 0 & 0 & (3,6) & 0 \\
* & * & * & -\gamma^{2} I & 0 & (4,6) & 0 \\
* & * & * & * & -I & 0 & 0 \\
* & * & * & * & * & -2 \varepsilon P & \varepsilon R \\
* & * & * & * & * & * & -R
\end{array}\right)<0
$$

where terms $(1,1),(1,2),(1,3),(1,4),(1,6),(2,2),(2,6),(3,6)$, and $(4,6)$ are given as in $(26)$. From the aforementioned discussion, we conclude the following result.

Proposition 2: For the given scalars $\tau>0, \gamma>0$, and $\varepsilon>0$, system (18) with $K_{\xi}=P^{-1} Y_{K}$ and $H_{\xi}=P^{-1} Y_{H}$ is globally asymptotically stable with an energy-gain bound $\gamma^{2}$ if there exist real $\left(n+n_{f}\right) \times\left(n+n_{f}\right)$ matrices $P>0, Q>0$, and $R>0$, a matrix $\Lambda=\operatorname{diag}\left(\lambda_{1}, \lambda_{2}, \ldots, \lambda_{m}\right)>0$, and matrices $Y_{K}$ and $Y_{H}$ with appropriate dimensions such that LMI (28) is satisfied.

Remark 1: For the given scalars $\tau>0$ and $\gamma>0$, a proper $\varepsilon>0$ is easily chosen by iteratively applying Proposition 2 . Similarly, for the given $\tau>0$ and $\varepsilon>0$ (or $\varepsilon>0$ and $\gamma>$ 0 , respectively), the minimum allowed $\gamma_{\min }$ (or the maximum allowed $\tau_{\max }$, respectively) can also be calculated by iteratively applying Proposition 2.

If the current state information is not available for measurement due to the existence of a time delay, one can set $K_{i}=$ $0(i=1,2)$. Then, the error system (18) becomes

$$
\left\{\begin{aligned}
\dot{\xi}(t)= & A_{\xi} \xi(t)-H_{\xi} D_{\xi} \xi(t-\tau) \\
& +B_{\xi} \eta(C e(t), y(t))+B_{\xi f} f_{0}(t) \\
z_{e}(t)= & F \xi(t) \quad \xi(\theta)=\phi_{\xi}(\theta) \quad \forall \theta \in[-\tau, 0] .
\end{aligned}\right.
$$
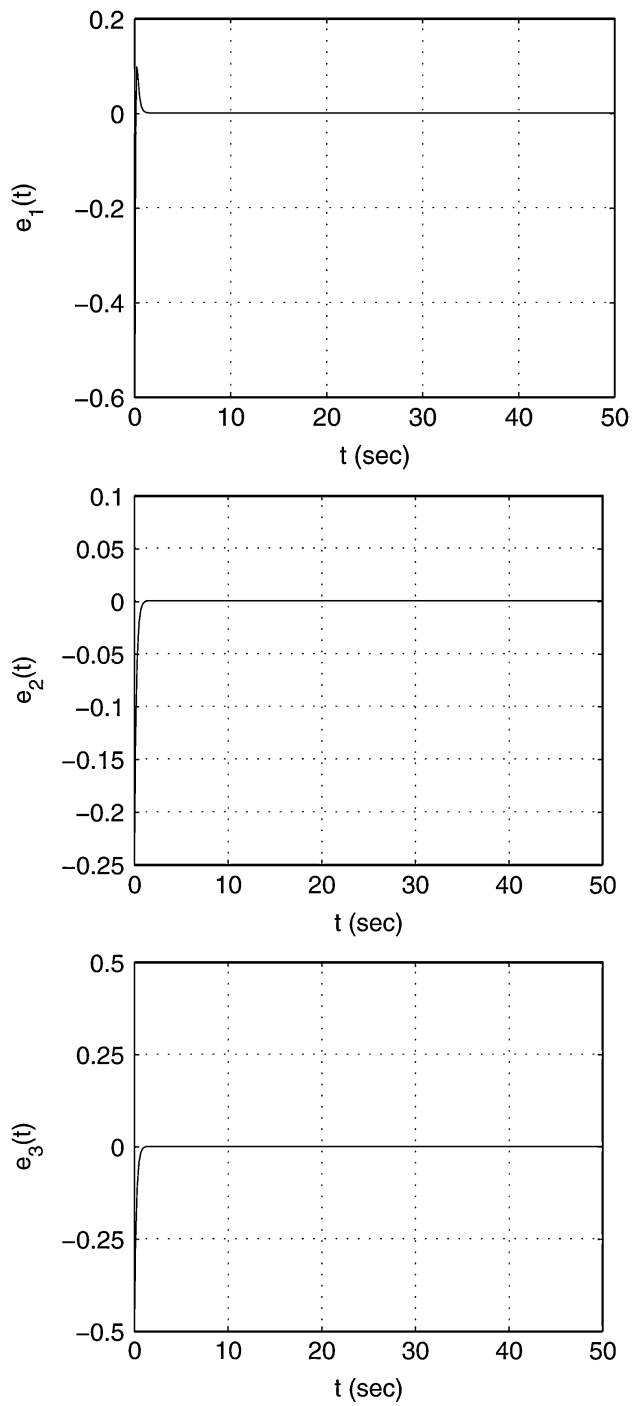

Fig. 4. Synchronization error without fault.

Proposition 2 implies the following result.

Corollary 3: For the given scalars $\tau>0, \gamma>0$, and $\varepsilon>$ 0 , system (29) with $H_{\xi}=P^{-1} Y_{H}$ is globally asymptotically stable with an energy-gain bound $\gamma^{2}$ if there exist real $\left(n+n_{f}\right) \times$ $\left(n+n_{f}\right)$ matrices $P>0, Q>0$, and $R>0$, a matrix $\Lambda=$ $\operatorname{diag}\left(\lambda_{1}, \lambda_{2}, \ldots, \lambda_{m}\right)>0$, and a matrix $Y_{H}$ with appropriate dimensions such that the LMI (28) with $Y_{K}=0$ is satisfied.

Similar to Proposition 2, we have the following delay-independent result using Corollary 1, which is also implied by Proposition 2.

Corollary 4: For a given scalar $\gamma>0$, system (18) with $K_{\xi}=P^{-1} Y_{K}$ and $H_{\xi}=P^{-1} Y_{H}$ is globally asymptotically stable with an energy-gain bound $\gamma^{2}$ if there exist real $(n+$ $\left.n_{f}\right) \times\left(n+n_{f}\right)$ matrices $P>0$ and $Q>0$, a matrix $\Lambda=$ $\operatorname{diag}\left(\lambda_{1}, \lambda_{2}, \ldots, \lambda_{m}\right)>0$, and matrices $Y_{K}$ and $Y_{H}$ with appropriate dimensions such that

$$
\left(\begin{array}{ccccc}
(1,1) & -Y_{H} D_{\xi} & P B_{\xi}+k C_{\xi}^{\mathrm{T}} \Lambda & P B_{\xi f} & F^{\mathrm{T}} \\
* & -Q & 0 & 0 & 0 \\
* & * & -2 \Lambda & 0 & 0 \\
* & * & * & -\gamma^{2} I & 0 \\
* & * & * & * & -I
\end{array}\right)<0
$$



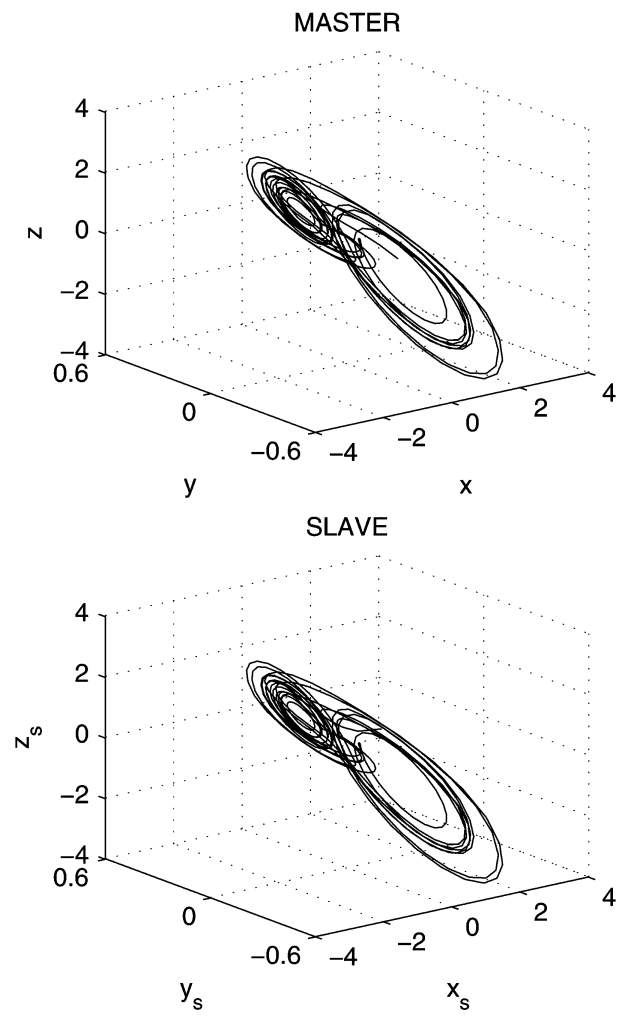

Fig. 5. Master-slave state with fault $f(t)$.

where

$$
(1,1)=A_{\xi}^{\mathrm{T}} P+P A_{\xi}+Y_{K} I_{0}+I_{0}^{\mathrm{T}} Y_{K}^{\mathrm{T}}+Q .
$$

If the fault $f(t)$ is not considered, we design feedback controller (3) such that error system (5) is globally asymptotically stable. Similar to Proposition 2, we have the following result applying Lemma 2 and Corollary 2.

Corollary 5: For a given scalar $\varepsilon_{1}>0$, system (5) with $K_{1}=$ $P_{1}^{-1} Y_{1 K}$ and $H_{1}=P_{1}^{-1} Y_{1 H}$ is globally asymptotically stable if there exist real $n \times n$ matrices $P_{1}>0, Q_{1}>0$, and $R_{1}>0$, a matrix $\Lambda_{1}=\operatorname{diag}\left(\lambda_{11}, \lambda_{12}, \ldots, \lambda_{1 m}\right)>0$, and matrices $Y_{1 K}$ and $Y_{1 H}$ with appropriate dimensions such that

$$
\left(\begin{array}{ccccc}
(1,1) & (1,2) & (1,3) & (1,4) & 0 \\
* & -Q_{1}-R_{1} & 0 & -\tau D^{\mathrm{T}} Y_{1 H}^{\mathrm{T}} & 0 \\
* & * & -2 \Lambda_{1} & \tau B^{\mathrm{T}} P_{1} & 0 \\
* & * & * & -2 \varepsilon_{1} P_{1} & \varepsilon_{1} R_{1} \\
* & * & * & * & -R_{1}
\end{array}\right)<0
$$

where

$$
\begin{aligned}
& (1,1)=A^{\mathrm{T}} P_{1}+P_{1} A+Y_{1 K}+Y_{1 K}^{\mathrm{T}}+Q_{1}-R_{1} \\
& (1,2)=-Y_{1 H} D+R_{1} \\
& (1,3)=P_{1} B+k C^{\mathrm{T}} \Lambda_{1} \\
& (1,4)=\tau\left(A^{\mathrm{T}} P_{1}+Y_{1 K}^{\mathrm{T}}\right) .
\end{aligned}
$$

\section{NUMERICAL EXAMPLE}

To show the effectiveness of the proposed fault-tolerant master-slave synchronization method, three kinds of faults are
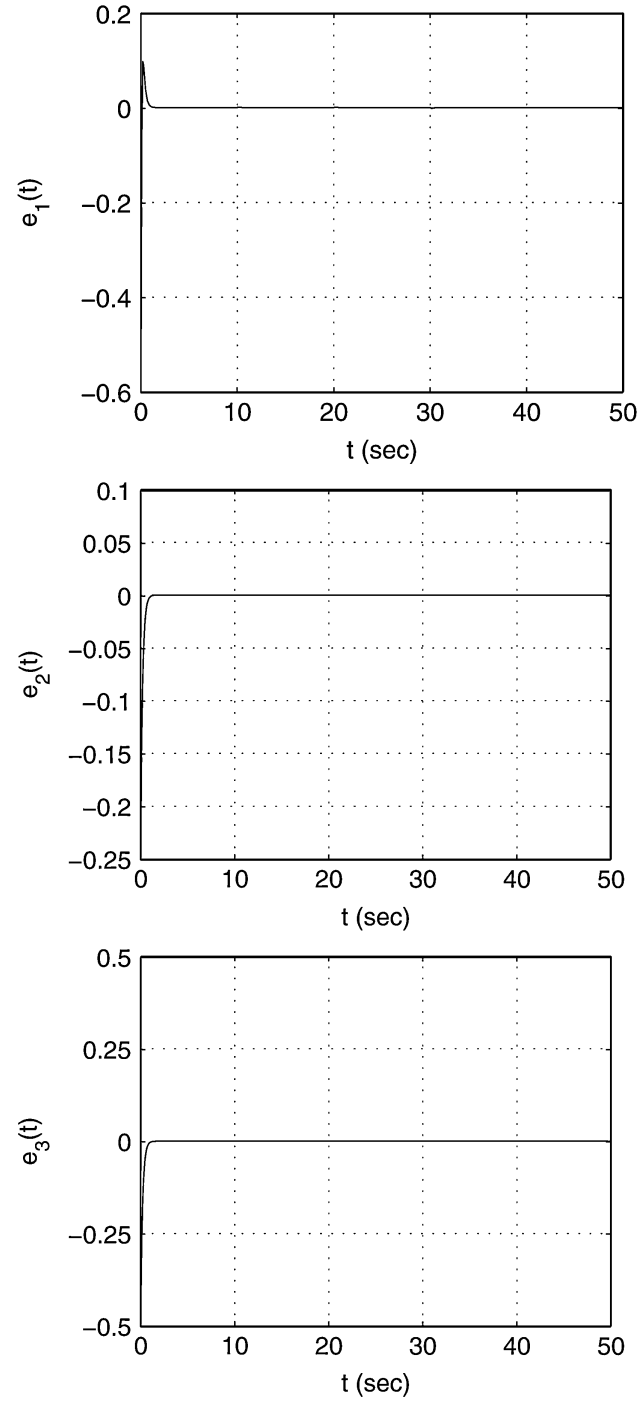

Fig. 6. Synchronization error with fault $f(t)$.

TABLE I

$\gamma_{\text {min }}$ FOR DIFFERENT TIME DELAYS $\tau$

\begin{tabular}{c|c|c|c|c|c}
\hline$\tau$ & 3.0 & 4.0 & 5.0 & 6.0 & 7.0 \\
\hline$\gamma_{\min }$ & 0.0779 & 0.1465 & 0.2486 & 0.4030 & 0.6539 \\
\hline
\end{tabular}

to be considered, i.e., the stepwise, slow-drifting, and sine-wave faults.

When a fault is taken into account, Chua's circuit in [5] and [20] becomes

$$
\left\{\begin{array}{l}
\dot{x}(t)=\alpha(y(t)-h(x(t)))+f(t) \\
\dot{y}(t)=x(t)-y(t)+z(t) \\
\dot{z}(t)=-\beta y(t)
\end{array}\right.
$$

where

$$
h(x(t))=m_{1} x(t)+\frac{1}{2}\left(m_{0}-m_{1}\right)(|x(t)+c|-|x(t)-c|)
$$



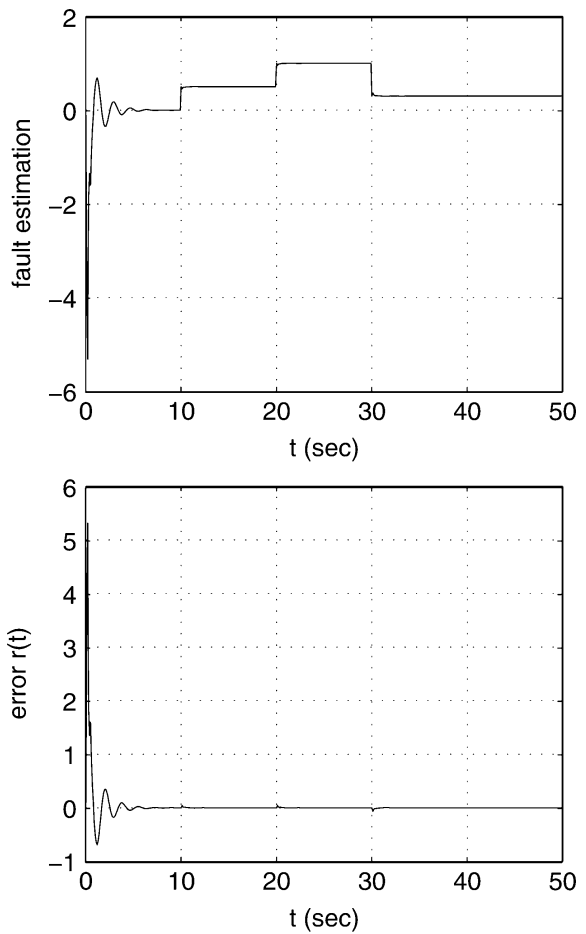

Fig. 7. Fault estimation $\hat{f}(t)$ and estimation error $r(t)$.
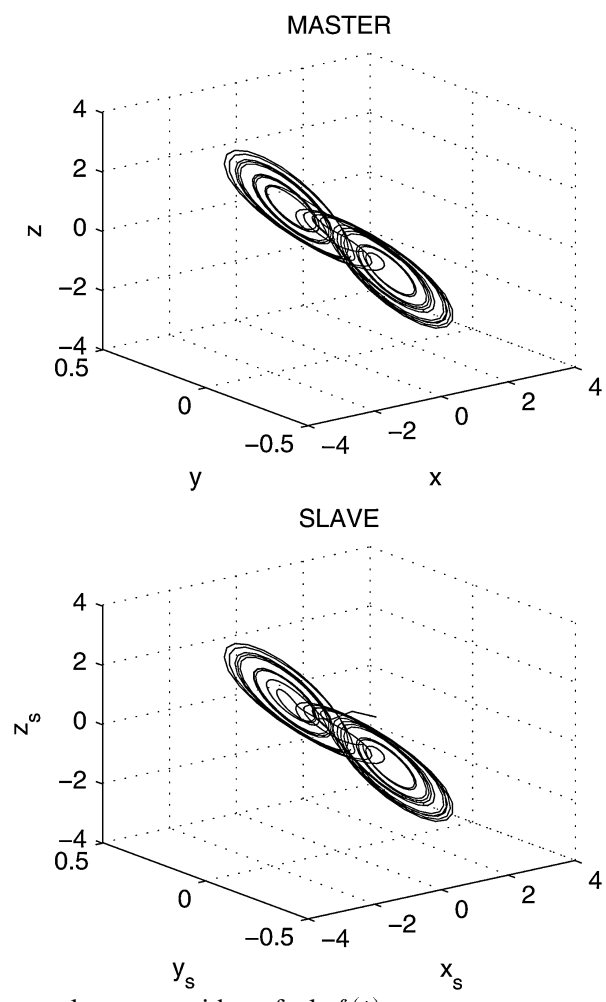

Fig. 8. Master-slave state without fault $f(t)$.

with $m_{0}=-(1 / 7), m_{1}=-(2 / 7), \alpha=9, \beta=14.28$, and $c=1$. Then, the master system can be represented in Lur'e form (7) with

$$
\begin{aligned}
& A=\left(\begin{array}{ccc}
-\alpha m_{1} & \alpha & 0 \\
1 & -1 & 1 \\
0 & -\beta & 0
\end{array}\right) \\
& B^{\mathrm{T}}=\left(\begin{array}{lll}
-\alpha\left(m_{0}-m_{1}\right) & 0 & 0
\end{array}\right) \\
& B_{f}^{\mathrm{T}}=C=D=\left(\begin{array}{lll}
1 & 0 & 0
\end{array}\right) \text {. }
\end{aligned}
$$
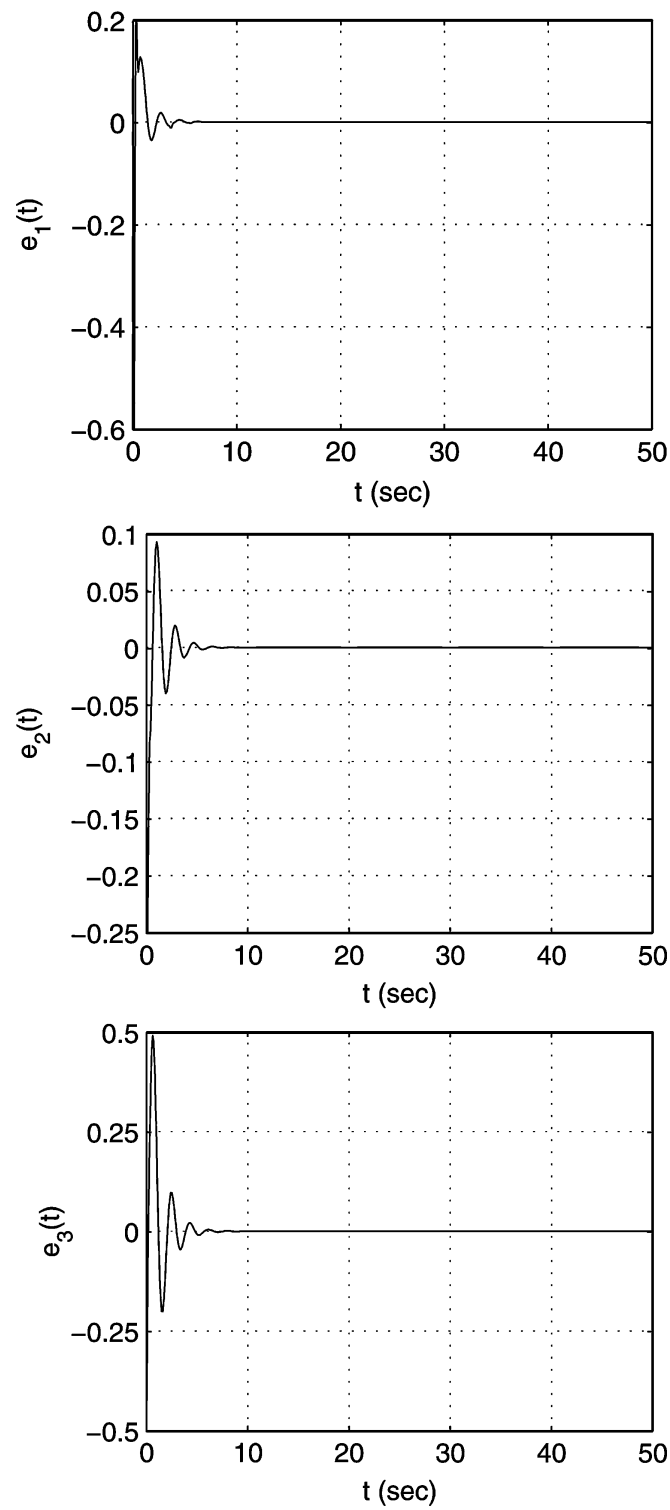

Fig. 9. Synchronization error without fault.

and $\varphi(\theta)=(1 / 2)(|\theta+c|-|\theta-c|)$ belonging to sector $[0, k]$ with $k=1$.

We first consider the stepwise fault characterized by (9) with $f_{0}(t)=\sum_{i=1}^{N} \rho_{i} \delta\left(t-t_{f i}\right)$. Set $F=I$. Choosing $\varepsilon=100$, applying Proposition 2, we calculate the minimum allowed $\gamma$ (i.e., $\left.\gamma_{\min }\right)$ and gain matrices $K_{i}$ and $H_{i}(i=1,2)$ for different time delays $\tau$. Table I lists the obtained $\gamma_{\min }$ for different time delays $\tau$.

For $\tau=5.0$ and $\gamma=0.3$, we have

$$
\begin{aligned}
K_{1} & =\left(\begin{array}{ccc}
-15.8524 & -9.0000 & -0.0000 \\
-1.0000 & -3.8663 & -1.0000 \\
0.0000 & 14.2800 & -4.8663
\end{array}\right) \\
K_{2} & =\left(\begin{array}{lll}
-67.4555 & 0.0000 & -0.0000
\end{array}\right) \\
H_{1}^{\mathrm{T}} & =\left(\begin{array}{lll}
0.5325 & 0.0000 & -0.0000
\end{array}\right) \times 10^{-3} \\
H_{2} & =0.0048 .
\end{aligned}
$$



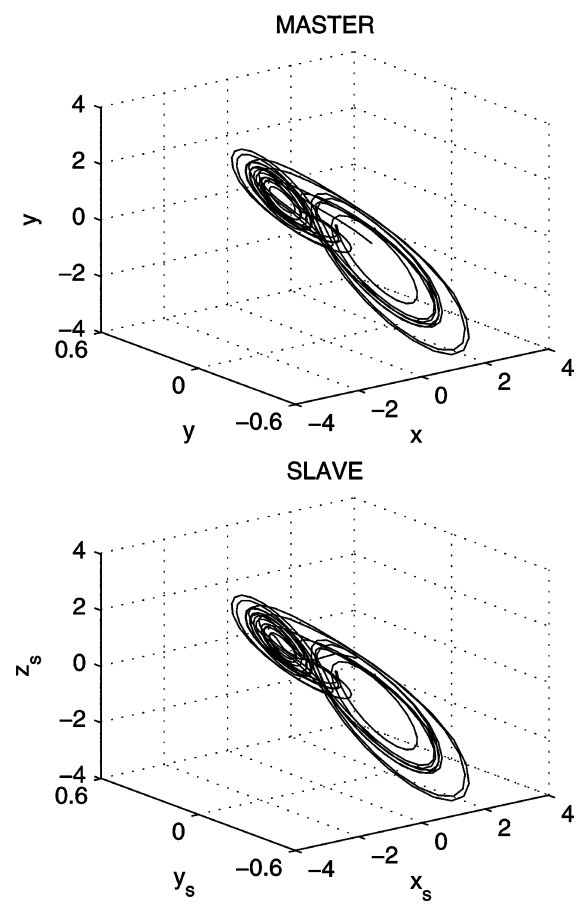

Fig. 10. Master-slave state with fault $f(t)$.

The initial conditions of the master and slave systems are chosen as

$$
\begin{aligned}
& \left(\begin{array}{lll}
x(0) & y(0) & z(0)
\end{array}\right)=\left(\begin{array}{lll}
-0.2 & -0.33 & 0.2
\end{array}\right) \\
& \left(\begin{array}{lll}
x_{s}(0) & y_{s}(0) & z_{s}(0)
\end{array}\right)=\left(\begin{array}{lll}
0.5 & -0.1 & 0.66
\end{array}\right) .
\end{aligned}
$$

Over time interval $[0,50] \mathrm{s}$, when a fault simulated as in Fig. 1 occurs, Fig. 2 shows the fault estimation $\hat{f}(t)$ and fault estimation error $r(t)$. Figs. 3-6 show the master system states $(x(t), y(t), z(t))$, the slave system states $\left(x_{s}(t), y_{s}(t), z_{s}(t)\right)$, and the synchronization errors $e_{1}(t)=x(t)-x_{s}(t)$, $e_{2}(t)=y(t)-y_{s}(t)$, and $e_{3}(t)=z(t)-z_{s}(t)$ for both the fault-free and faulty cases, respectively.

If the current master-slave system states are not available, we set $K_{i}=0(i=1,2)$. Choosing $\varepsilon=0.1$, applying Corollary 1 , we calculate the minimum allowed $\gamma_{\min }$ and gain matrices $H_{i}(i=1,2)$ for different time delays $\tau$. Table II lists the obtained $\gamma_{\min }$ for different time delays $\tau$.

For $\tau=0.05$ and $\gamma=0.5282$, we have

$$
\begin{aligned}
H_{1}^{\mathrm{T}} & =\left(\begin{array}{lll}
10.6210 & 1.8306 & -1.2025
\end{array}\right) \\
H_{2} & =25.4730 .
\end{aligned}
$$

Similar to the case of $K_{i} \neq 0(i=1,2)$, the fault estimation and estimation error, the master-slave system state, and the synchronization errors for both the fault-free and faulty cases are shown in Figs. 7-11, respectively.

In order to compare the obtained result with that of the master-slave synchronization scheme $\mathcal{M}_{0}-\mathcal{S}-\mathcal{C}_{0}$, we choose $H_{1}^{\mathrm{T}}=\left(\begin{array}{lll}10.6210 & 1.8306 & -1.2025\end{array}\right)$ and $K_{1}=0$. When a fault in Fig. 1 occurs, the master-slave synchronization error using $\mathcal{M}_{0}-\mathcal{S}-\mathcal{C}_{0}$ is shown in Fig. 12. It is clear to see from Figs. 11 and 12 that, when a fault in Fig. 1 occurs, the master-slave synchronization has been realized using the new
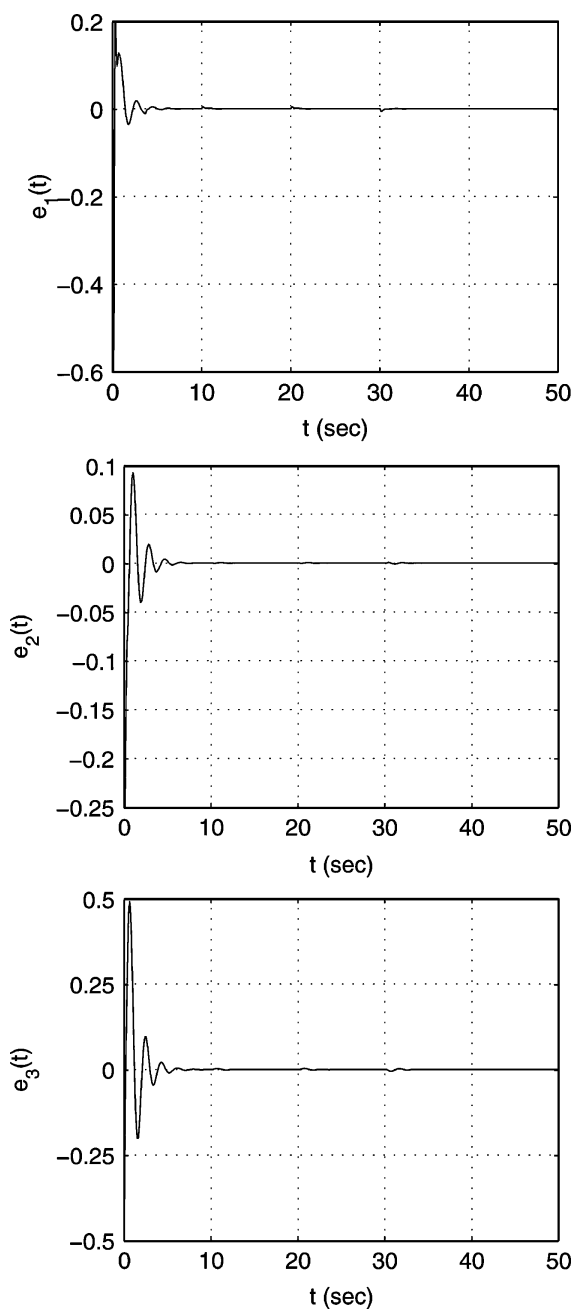

Fig. 11. Synchronization error with fault $f(t)$.

TABLE II $\gamma_{\min }$ FOR DIFFERENT TIME DELAYS $\tau$

\begin{tabular}{c|c|c|c|c|c}
\hline$\tau$ & 0.02 & 0.04 & 0.06 & 0.08 & 0.10 \\
\hline$\gamma_{\min }$ & 0.3005 & 0.4075 & 0.5553 & 0.7704 & 1.1335 \\
\hline
\end{tabular}

scheme $\mathcal{M}-\mathcal{S}-\mathcal{C}-\mathcal{F}$, but that cannot be achieved by scheme $\mathcal{M}_{0}-\mathcal{S}-\mathcal{C}_{0}$.

Next, we consider the slow-drifting fault described by (10) with $f_{0}(t)=\sum_{i=1}^{N} \rho_{i} \delta\left(t-t_{f i}\right)$. Similar to the case of the stepwise fault, we can calculate the minimum allowed $\gamma$ and gain matrices $K_{i}, H_{i}(i=1,2)$ for different time delays $\tau$ applying Proposition 2. For the sake of simplicity, these are not listed here. Let $\varepsilon=100, \tau=5.0$, and $\gamma=0.4$. We have

$$
\begin{aligned}
K_{1} & =\left(\begin{array}{ccc}
-21.9598 & -9.0000 & -0.0000 \\
-1.0000 & -3.8256 & -1.0000 \\
0.0000 & 14.2800 & -4.8256
\end{array}\right) \\
K_{2} & =\left(\begin{array}{ccc}
-507.4973 & 0.0001 & -0.0002 \\
-180.2822 & 0.0000 & -0.0001
\end{array}\right) \\
H_{1}^{\mathrm{T}} & =\left(\begin{array}{lll}
0.2458 & 0.0000 & -0.0000
\end{array}\right) \times 10^{-3} \\
H_{2} & =\left(\begin{array}{l}
0.0211 \\
0.0045
\end{array}\right) .
\end{aligned}
$$



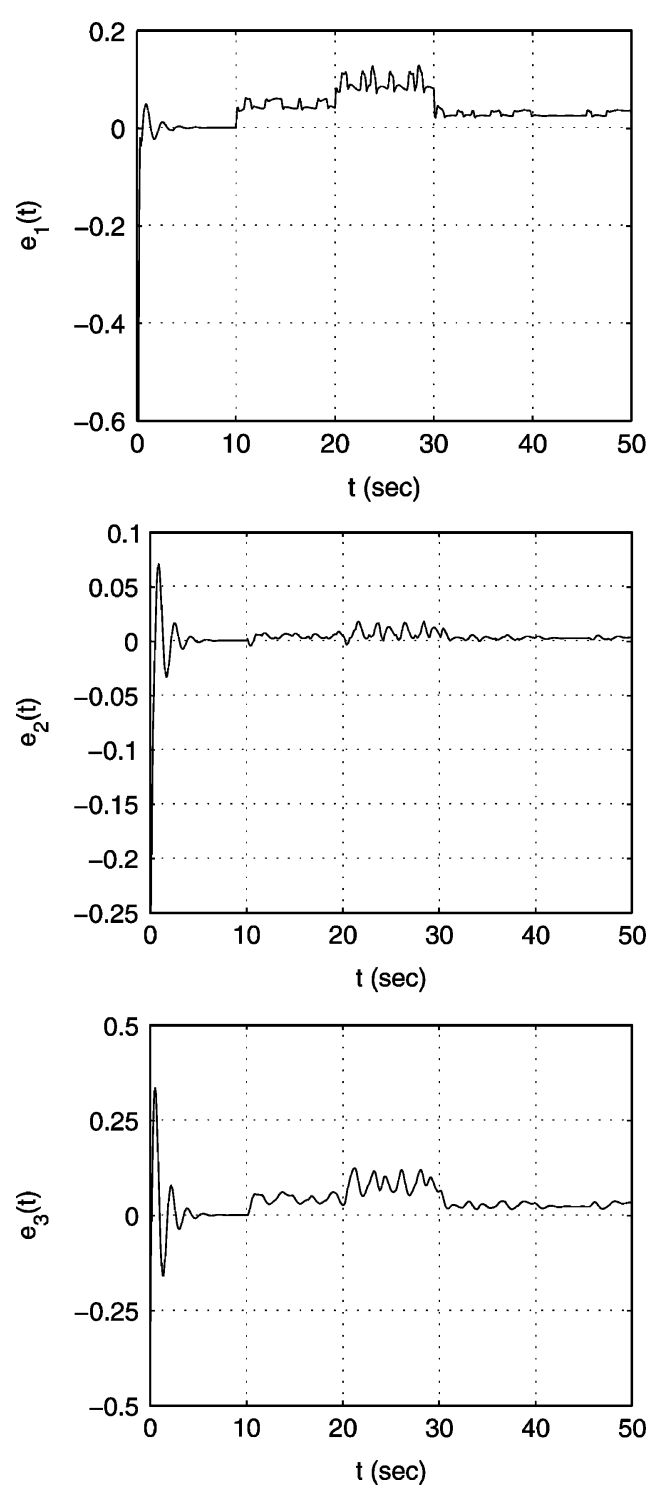

Fig. 12. Synchronization error with fault $f(t)\left(\mathcal{M}_{0}-\mathcal{S}-\mathcal{C}_{0}\right)$.

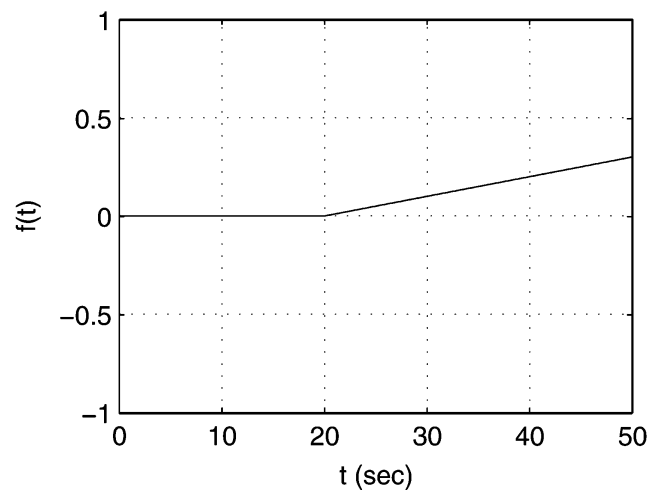

Fig. 13. Ramp fault signal $f(t)$.

When a ramp fault in Fig. 13 occurs, Fig. 14 shows the fault estimation $\hat{f}(t)$ and fault estimation error $r(t)$. Figs. 15 and 16 show the faulty-case master system states $(x(t), y(t), z(t))$, the
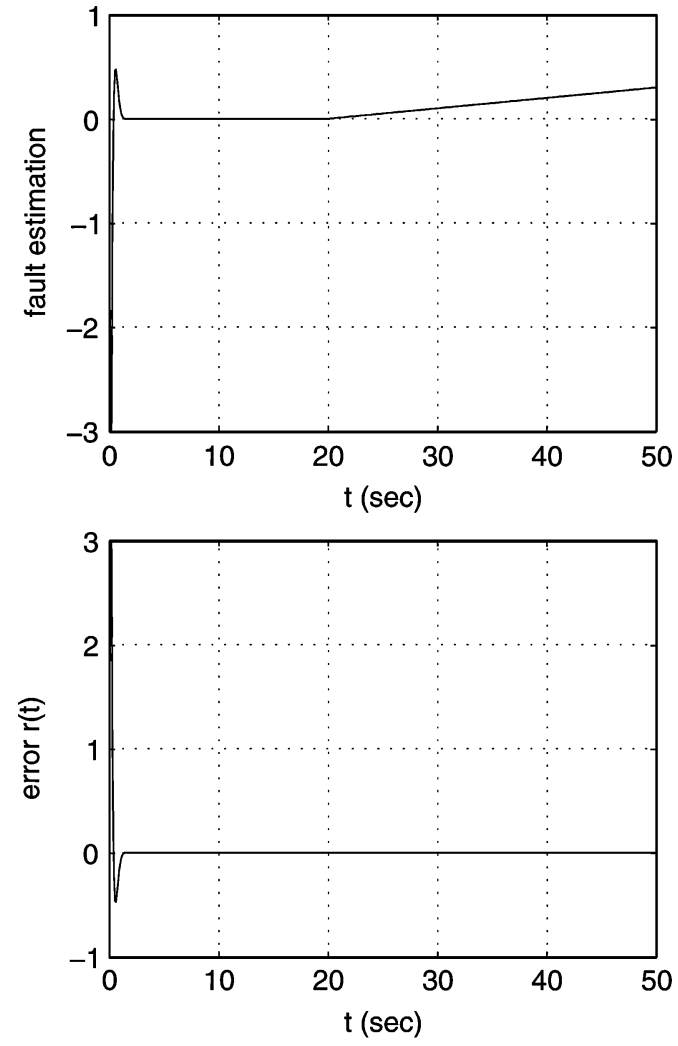

Fig. 14. Fault estimation $\hat{f}(t)$ and estimation error $r(t)$.
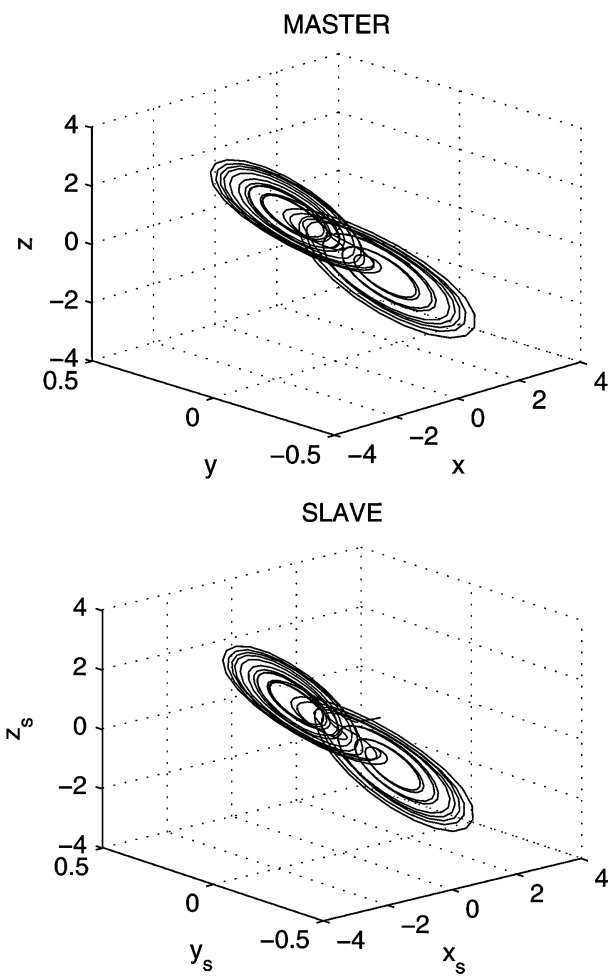

Fig. 15. Master-slave state with fault $f(t)$.

slave system states $\left(x_{s}(t), y_{s}(t), z_{s}(t)\right)$, and the synchronization errors $e_{1}(t), e_{2}(t)$, and $e_{3}(t)$, respectively.

Finally, we consider the sine-wave fault given by (11) 

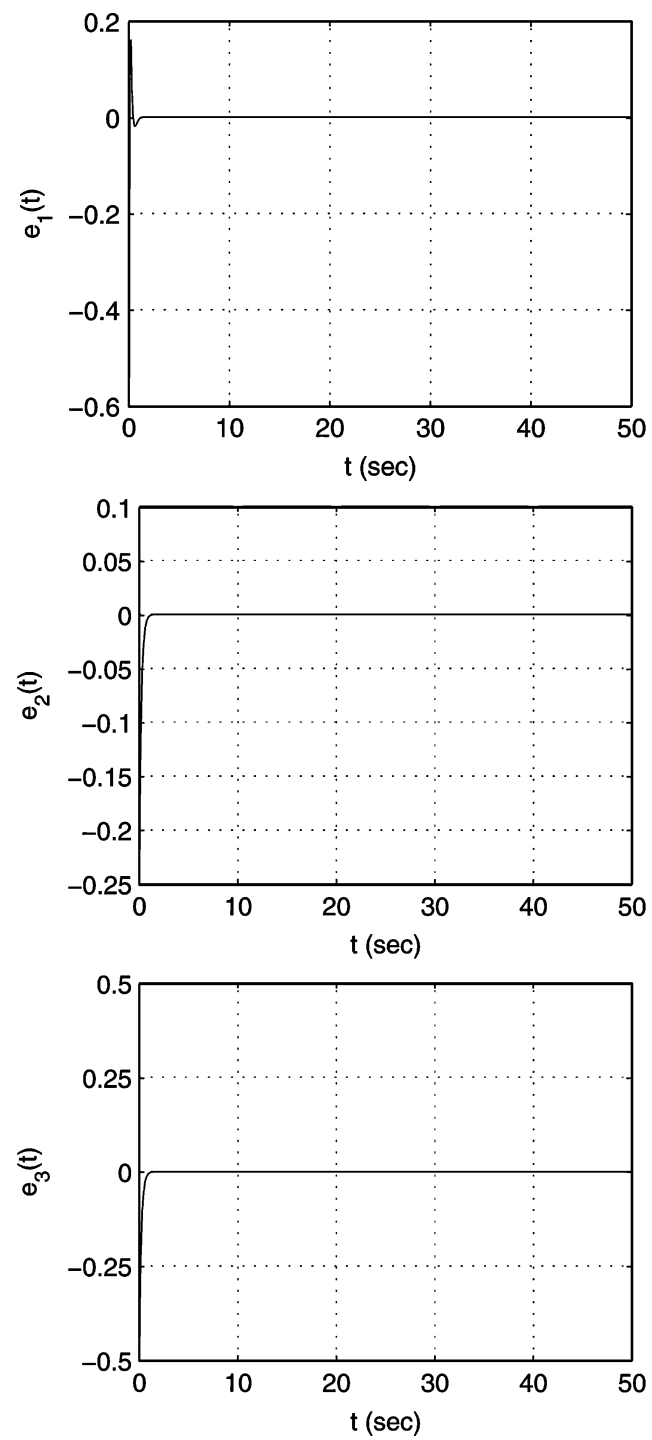

Fig. 16. Synchronization error with fault $f(t)$.

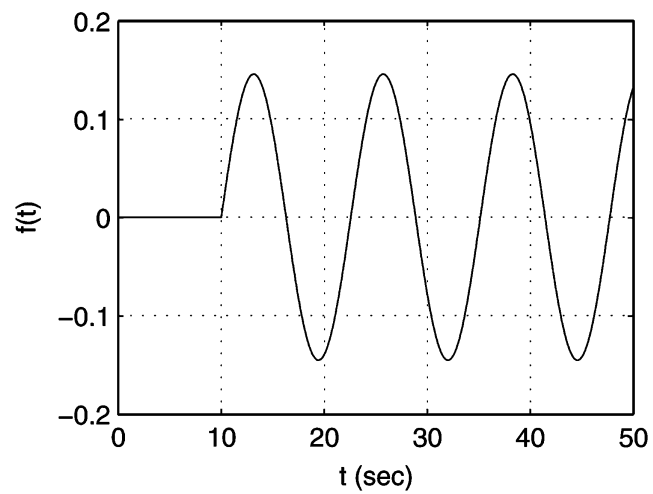

Fig. 17. Sine-wave-fault signal $f(t)$.

with $f_{0}(t)=\sum_{i=1}^{N} \rho_{i} \delta\left(t-t_{f i}\right)$. Similarly, we have

$$
K_{1}=\left(\begin{array}{ccc}
-15.7950 & -9.0000 & -0.0000 \\
-1.0000 & -3.8300 & -1.0000 \\
0.0000 & 14.2800 & -4.8300
\end{array}\right)
$$
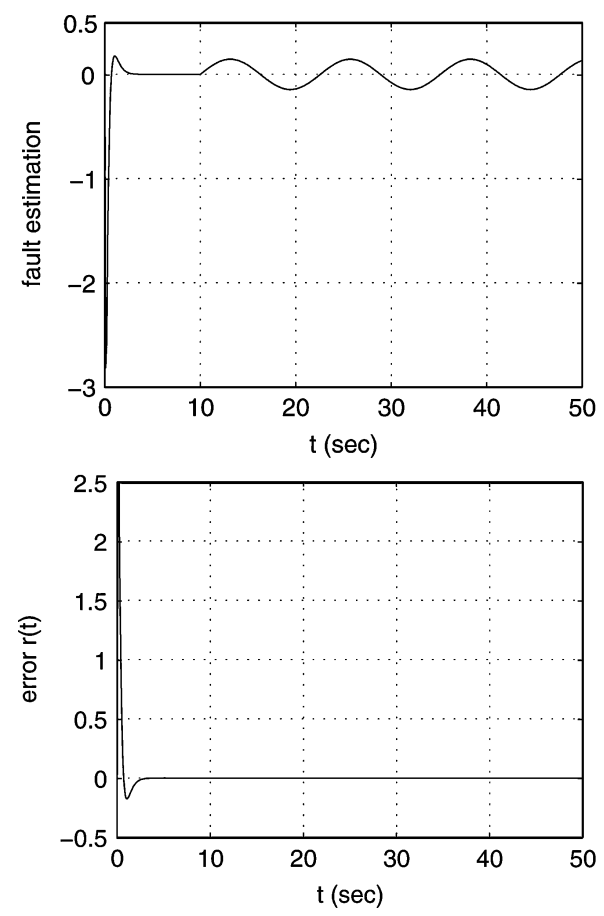

Fig. 18. Fault estimation $\hat{f}(t)$ and estimation error $r(t)$.
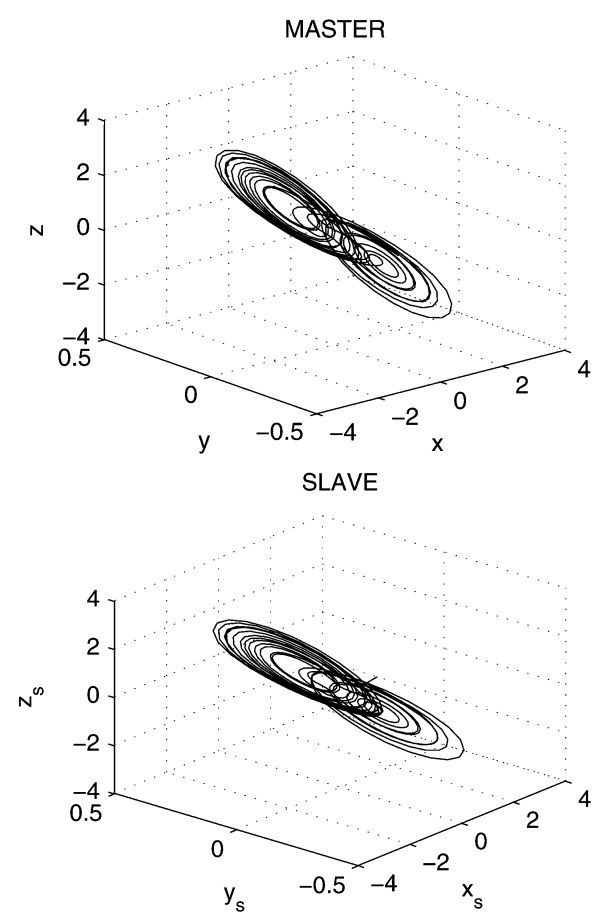

Fig. 19. Master-slave state with fault $f(t)$.

$$
\begin{aligned}
K_{2} & =\left(\begin{array}{lll}
-100.3358 & 0.0000 & -0.0000 \\
-81.0135 & 0.0000 & -0.0000
\end{array}\right) \\
H_{1}^{\mathrm{T}} & =\left(\begin{array}{lll}
0.0013 & 0.0000 & -0.0000
\end{array}\right) \\
H_{2} & =\left(\begin{array}{l}
0.0460 \\
0.0174
\end{array}\right)
\end{aligned}
$$

for $\varepsilon=100, \tau=5.0$, and $\gamma=1.1$. A sine-wave fault $f(t)$, the fault estimation $\hat{f}(t)$, fault estimation error $r(t)$, faulty-case master system states $(x(t), y(t), z(t))$, slave system 

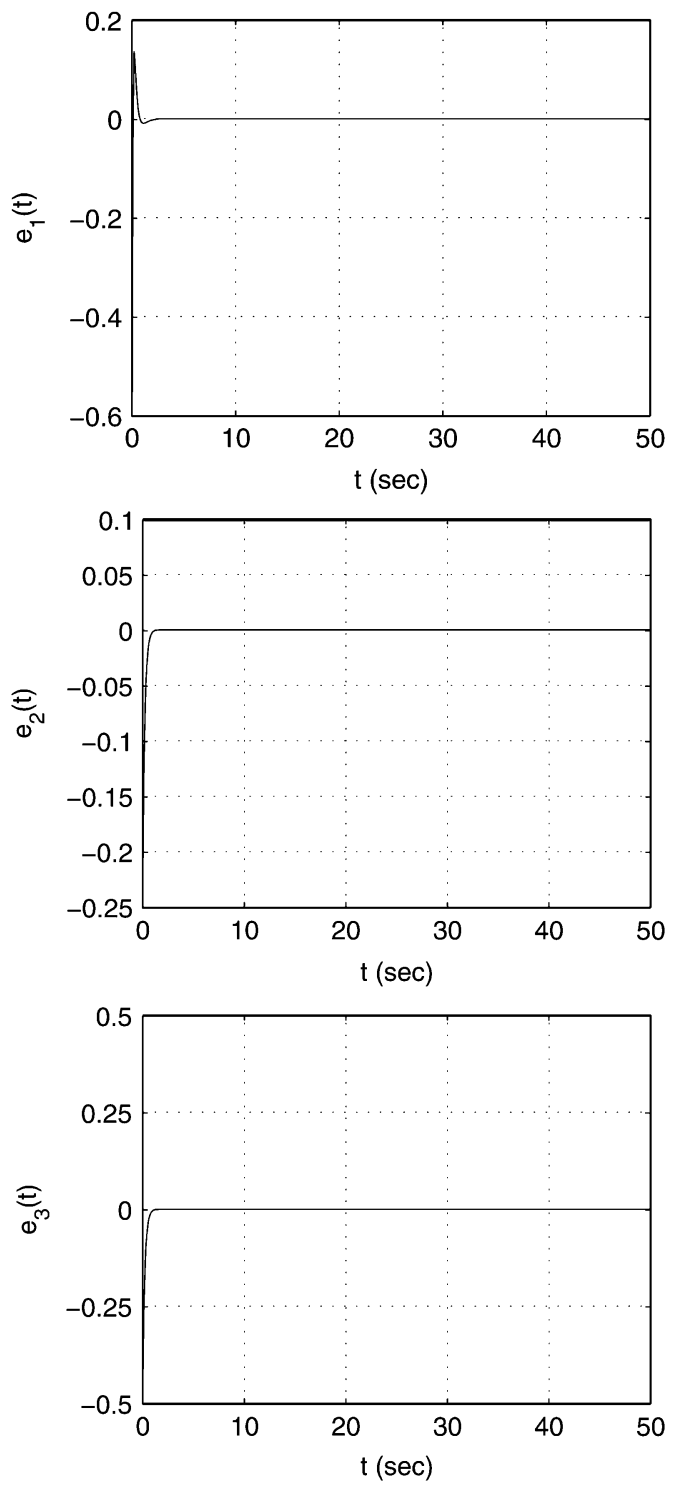

Fig. 20. Synchronization error with fault $f(t)$.

states $\left(x_{s}(t), y_{s}(t), z_{s}(t)\right)$, and the corresponding synchronization errors $e_{1}(t), e_{2}(t)$, and $e_{3}(t)$ are shown in Figs. 17-20, respectively.

\section{CONCLUSION}

The fault-tolerant master-slave synchronization for Lur'e systems using time-delay feedback has been addressed. First, an observer-based fault estimator has been used to estimate faults in the master system, and the fault estimation has been incorporated into the feedback controller. Then, the fault-tolerant master-slave synchronization has been formulated so as to discuss the global asymptotic stability of the synchronization error system and the bound of energy gain from an exogenous signal to synchronization and fault estimation errors. New delay-dependent criteria have been derived to analyze the master-slave synchronization error system, and some existing results have been covered as their special cases. Based on these analysis results, delay-dependent sufficient conditions on the existence of a fault-tolerant controller and a solution to the controller and fault-estimator gain matrices have been obtained in terms of LMIs. Finally, a Chua's circuit has shown the effectiveness of the proposed method, and the simulation results have shown that the master-slave synchronization has been achieved for Lur'e systems by using the fault-tolerant feedback control with time delay.

\section{REFERENCES}

[1] J. Cao, H. X. Li, and D. W. C. Ho, "Synchronization criteria of Lur'e systems with time-delay feedback control," Chaos, Solitons Fractals, vol. 23 , no. 4, pp. 1285-1298, Feb. 2005.

[2] G. Chen and X. Dong, From Chaos to Order-Perspectives, Methodologies, and Applications. Singapore: World Scientific, 1998.

[3] G. Chen and M. J. Ogorzalek, Eds., "Special issue on control and synchronization of chaos," Int. J. Bifurc. Chaos, vol. 10, no. 3/4, 2000.

[4] W. Chen and M. Saif, "An iterative learning observer for fault detection and accommodation in nonlinear time-delay systems," Int. J. Robust Nonlinear Control, vol. 16, no. 1, pp. 1-19, Jan. 2006.

[5] L. O. Chua, M. Komura, and T. Matsumoto, "The double scroll family," IEEE Trans. Circuits Syst. I, Fundam. Theory Appl., vol. CAS1-33, no. 11, pp. 1072-1118, Nov. 1986

[6] C. Cruz-Hernández, "Synchronization of time-delay Chua's oscillator with application to secure communication," Nonlinear Dyn. Syst. Theory, vol. 4, no. 1, pp. 1-13, 2004.

[7] P. F. Curran and L. O. Chua, "Absolute stability theory and the synchronization problem,” Int. J. Bifurc. Chaos, vol. 7, no. 6, pp. 1375-1382, Jun. 1997.

[8] P. F. Curran, J. A. K. Suykens, and L. O. Chua, "Absolute stability theory and master-slave synchronization problem," Int. J. Bifurc. Chaos, vol. 7, no. 12, pp. 2891-2896, Dec. 1997.

[9] S. X. Ding, Model-Based Fault Diagnosis Technique. Berlin, Germany: Springer-Verlag, 2008.

[10] U. Feldmann, M. Hasler, and W. Schwarz, "Communication by chaotic signals: The inverse system approach," Int. J. Circuit Theory Appl., vol. 24, no. 5, pp. 551-579, 1996.

[11] L. Guo and H. Wang, "Fault detection and diagnosis for general stochastic systems using B-spline expansions and nonlinear filters,' IEEE Trans. Circuits Syst. I, Fundam. Theory Appl., vol. 52, no. 8, pp. 1644-1652, Aug. 2005.

[12] K. Gu, V. L. Kharitonov, and J. Chen, Stability of Time-Delay Systems. Cambridge, MA: Birkhäuser, 2003.

[13] Q.-L. Han, "New delay-dependent synchronization criteria for Lur'e systems using time delay feedback control," Phys. Lett. A, vol. 360, no. 4/5, pp. 563-569, Jan. 2007.

[14] Q.-L. Han, "On designing time-varying delay feedback controllers for master-slave synchronization of Lur'e systems," IEEE Trans. Circuits Syst. I, Fundam. Theory Appl., vol. 54, no. 7, pp. 1573-1583, Jul. 2007.

[15] Q.-L. Han, "A new delay-dependent stability criterion for linear neutral systems with norm-bounded uncertainties in all system matrices," Int. J. Syst. Sci., vol. 36, no. 8, pp. 469-475, Aug. 2005.

[16] G. P. Jiang and G. Chen, "A state-observer-based approach for synchronization in complex dynamical networks," IEEE Trans. Circuits Syst. I, Fundam. Theory Appl., vol. 53, no. 12, pp. 2739-2745, Dec. 2006.

[17] M. P. Kennedy and M. J. Ogorzalek, Eds., "Special issue on chaos synchronization and control: Theory and applications," IEEE Trans. Circuits Syst. I, Fundam. Theory Appl., vol. 44, no. 10, pp. 853-854, 1997.

[18] X. Liao and G. Chen, "Chaos synchronization of general Lur'e systems via time-delay feedback control," Int. J. Bifurc. Chaos, vol. 13, no. 1, pp. 207-213, Jan. 2003.

[19] B. M. Mirkin and P. O. Gutman, "Model reference adaptive control of state delayed systems with actuator failures," Int. J. Control, vol. 78, no. 3, pp. 186-195, Feb. 2005

[20] R. N. Madan, Chua Circuit: A Paradigm for Chaos. Singapore: World Scientific, 1993.

[21] H. Nijmeijer and I. M. Y. Mareels, "An observer looks at synchronization," IEEE Trans. Circuits Syst. I, Fundam. Theory Appl., vol. 44, no. 10, pp. 882-890, Oct. 1997.

[22] L. M. Pecora and T. L. Carroll, "Synchronization in chaotic systems," Phys. Rev. Lett., vol. 64, no. 8, pp. 821-824, Feb. 1990.

[23] H. Sira-Ramirez and C. Cruz-Hernandez, "Synchronization of chaotic systems: A generalized Hamiltonian systems approach," Int. J. Bifurc. Chaos, vol. 11, no. 5, pp. 1381-1395, May 2001. 
[24] J. A. K. Suykens, P. F. Curran, and L. O. Chua, "Robust nonlinear $H_{\infty}$ synchronization of chaotic Lur'e systems," IEEE Trans. Circuits Syst. I, Fundam. Theory Appl., vol. 44, no. 10, pp. 891-904, Oct. 1997.

[25] J. A. K. Suykens, P. F. Curran, and L. O. Chua, "Robust synthesis for master-slave synchronization of Lur'e systems," IEEE Trans. Circuits Syst. I, Fundam. Theory Appl., vol. 46, no. 7, pp. 841-850, Jul. 1999.

[26] H. Wang, J. Lam, S. Xu, and S. Huang, "Robust $H_{\infty}$ reliable control for a class of uncertain neutral delay systems," Int. J. Syst. Sci., vol. 33, no. 7, pp. 611-622, Jun. 2002.

[27] C. W. Wu and L. O. Chua, "A unified framework for synchronization and control of dynamical systems," Int. J. Bifurc. Chaos, vol. 4, no. 4, pp. 979-989, Aug. 1994.

[28] T. Zhang and G. Feng, "Output tracking of piecewise-linear systems via error feedback regulator with application to synchronization of nonlinear Chua's circuit," IEEE Trans. Circuits Syst. I, Fundam. Theory Appl., vol. 54, no. 8, pp. 1644-1652, Aug. 2007.

[29] D. Yue, J. Lam, and D. W. C. Ho, "Reliable $H_{\infty}$ control of uncertain descriptor systems with multiple time delays," Proc. Inst. Elect. Eng. —Control Theory Appl., vol. 150, no. 6, pp. 557-564, Nov. 2003.

[30] M. E. Yalcin, J. A. K. Suykens, and J. Vandewalle, "Master-slave synchronization of Lur'e systems with time-delay," Int. J. Bifurc. Chaos, vol. 11, no. 6, pp. 1707-1722, Jun. 2001.

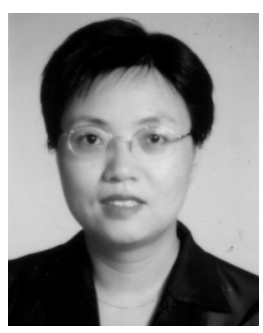

Maiying Zhong received the B.S. and M.S. degrees from Shandong University, Jinan, China, in 1986 and 1989, respectively, and the Ph.D. degree from the Northeastern University, Shenyang, China, in 1999.

From November 2000 to January 2002, she was a Visiting Scholar at the University of Applied Sciences Lausitz, Brandenburg, Germany. From March 2002 to July 2008, she was a Professor with the School of Control Science and Engineering, Shandong University. From April 2006 to March 2007, she was a Postdoctoral Researcher Fellow with the Central Queensland University, Rockhampton, Australia, where she is currently with the Centre for Intelligent and Networked Systems. In September 2008, she joined Beihang University, Beijing, China, where she is currently a Professor with the School of Instrument Science and Opto-Electronics Engineering. Her research interests include fault detection and fault-tolerant control.

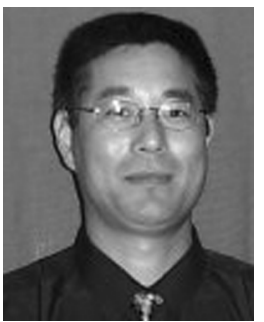

Qing-Long Han received the B.Sc. degree in mathematics from Shandong Normal University, Jinan, China, in 1983 and the M.Eng. and Ph.D. degrees in information science (electrical engineering) from the East China University of Science and Technology, Shanghai, China, in 1992 and 1997, respectively.

From September 1997 to December 1998, he was a Postdoctoral Researcher Fellow with the Laboratoire d'Automatique et d'Informatique Industrielle, Ecole Supérieure d'Ingénieurs de Poitiers (LAII-ESIP), Université de Poitiers, Poitiers, France. From January 1999 to August 2001, he was a Research Assistant Professor with the Department of Mechanical and Industrial Engineering, Southern Illinois University, Edwardsville. In September 2001, he joined Central Queensland University, Rockhampton, Australia, where he is currently a Professor with the School of Computing Sciences and an Associate Dean (Research and Innovation) with the Faculty of Arts, Business, Informatics and Education and where he is also with the Centre for Intelligent and Networked Systems. He has held a Visiting Professor position with LAII-ESIP, Université de Poitiers, a Chair Professor position with Hangzhou Dianzi University, Hangzhou, China, as well as a Guest Professor position in three Chinese universities. His research interests include time-delay systems, robust control, networked control systems, neural networks, complex systems, and software development processes. He has published over 150 refereed papers in technical journals and conference proceedings. 